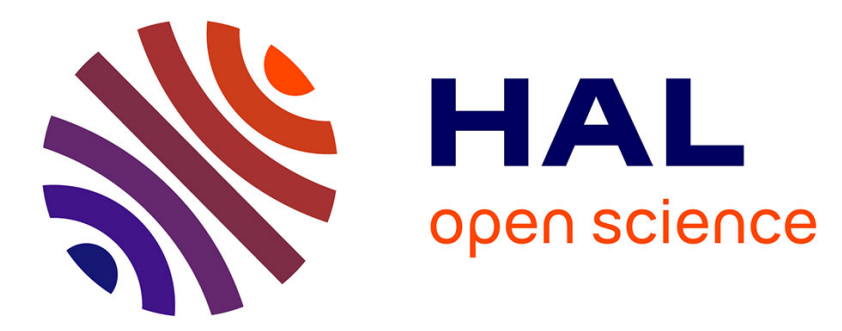

\title{
Testing Sign Conditions on a Multivariate Polynomial and Applications
}

Mohab Safey El Din

\section{To cite this version:}

Mohab Safey El Din. Testing Sign Conditions on a Multivariate Polynomial and Applications. [Research Report] RR-5995, INRIA. 2006. inria-00105835v2

\section{HAL Id: inria-00105835 https://hal.inria.fr/inria-00105835v2}

Submitted on 16 Oct 2006

HAL is a multi-disciplinary open access archive for the deposit and dissemination of scientific research documents, whether they are published or not. The documents may come from teaching and research institutions in France or abroad, or from public or private research centers.
L'archive ouverte pluridisciplinaire HAL, est destinée au dépôt et à la diffusion de documents scientifiques de niveau recherche, publiés ou non, émanant des établissements d'enseignement et de recherche français ou étrangers, des laboratoires publics ou privés. 
INSTITUT NATIONAL DE RECHERCHE EN INFORMATIQUE ET EN AUTOMATIQUE

\title{
Testing Sign Conditions on a Multivariate Polynomial and Applications
}

\author{
Mohab Safey El Din
}

\section{$\mathbf{N}^{\circ} \mathbf{5 9 9 5}$}

Octobre 2006

Thème SYM 



\title{
Testing Sign Conditions on a Multivariate Polynomial and Applications
}

\author{
Mohab Safey El Din* \\ Thème SYM — Systèmes symboliques \\ Projets SALSA \\ Rapport de recherche $\mathrm{n}^{\circ} 5995$ - Octobre 2006 - 34 pages
}

\begin{abstract}
Let $f$ be a polynomial in $\mathbb{Q}\left[X_{1}, \ldots, X_{n}\right]$ of degree $D$. We focus on testing the emptiness and computing at least one point in each connected component of the semialgebraic set defined by $f>0$ (or $f<0$ or $f \neq 0$ ). To this end, the problem is reduced to computing at least one point in each connected component of a hypersurface defined by $f-e=0$ for $e \in \mathbb{Q}$ positive and small enough. We provide an algorithm allowing us to determine a positive rational number $e$ which is small enough in this sense. This is based on the efficient computation of the set of generalized critical values of the mapping $f: y \in \mathbb{C}^{n} \rightarrow f(y) \in \mathbb{C}$ which is the union of the classical set $K_{0}(f)$ of critical values of the mapping $f$ and $K_{\infty}(f)$ of asymptotic critical values of the mapping $f$. Then, we show how to use the computation of generalized critical values in order to obtain an efficient algorithm deciding the emptiness of a semi-algebraic set defined by a single inequality or a single inequation. At last, we show how to apply our contribution to determining if a hypersurface contains real regular points. We provide complexity estimates for probabilistic versions of the latter algorithms which are within $\mathcal{O}\left(n^{7} D^{4 n}\right)$ arithmetic operations in $\mathbb{Q}$. The paper ends with practical experiments showing the efficiency of our approach.
\end{abstract}

Key-words: computer algebra, polynomial system solving, inequalities, real solutions, generalized critical values

* Mohab.Saf ey@lip6.fr

Unité de recherche INRIA Rocquencourt

Domaine de Voluceau, Rocquencourt, BP 105, 78153 Le Chesnay Cedex (France)

Téléphone : +33139635511-Télécopie : +331396353 30 


\section{Algorithme efficace pour tester le signe d'un polynôme univarié et ses applications}

Résumé : Soit $f$ un polynôme dans $\mathbb{Q}\left[X_{1}, \ldots, X_{n}\right]$ de degré $D$. On s'intéresse au problème de tester le vide d'un ensemble semi-algébrique défini par $f>0$ (ou $f<0$ ou $f \neq 0$ ). Pour ce faire, on réduit le problème au calcul d'au moins un point par composante connexe d'une hypersurface définie par $f-e=0$ pour $e \in \mathbb{Q}$ positif et suffisamment petit. On donne un algorithme permettant de déterminer un rationnel positif $e$ qui est en ce sens suffisamment petit. Cet algorithme est basé sur le calcul efficace de valeurs critiques généralisées de l'application polynomiale $f: y \in \mathbb{C}^{n} \rightarrow f(y) \in \mathbb{C}$ qui l'union de l'ensemble classique $K_{0}(f)$ des valeurs critiques de $f$ et de l'ensemble $K_{\infty}(f)$ des valeurs critiques asymptotiques de l'application $f$. Cet algorithme effectue le calcul en $\mathcal{O}\left(n^{7} D^{4 n}\right)$ opérations arithmétiques dans $\mathbb{Q}$. Puis on montre comment utiliser ce calcul de valeurs critiques généralisées pour obtenir un algorithme efficace décidant du vide (ou calculant au moins un point par composante connexe) d'un semi-algébrique défini par une seule inégalité ou inéquation. Enfin, on montre comment appliquer cette contribution au problème de déterminer si une hypersurface contient au moins un point réel régulier. Cet article se termine par des résultats expérimentaux montrant l'efficacité pratique de notre approche.

Mots-clés : calcul formel, résolution de systèmes polynomiaux, inégalités, solutions réelles, valeurs critiques généralisées 


\section{Introduction}

Let $f$ be a polynomial in $\mathbb{Q}\left[X_{1}, \ldots, X_{n}\right]$ of degree $D$ and $\mathcal{S}_{+} \subset \mathbb{R}^{n}$ (resp. $\mathcal{S}_{-}$and $\mathcal{S}$ ) be the semi-algebraic set defined by $f>0$ (resp. $f<0$ and $f \neq 0$ ). The aim of this paper is to provide an efficient algorithm in practice which computes at least one point in each connected component of $\mathcal{S}$ (resp. $\mathcal{S}_{-}$and $\mathcal{S}$ ).

This question is of first importance since solving parametric polynomial systems of equations and inequalities is reduced to compute at least one point in each connected component of the complementary of a real hypersurface (see [28]). This question also appears as a black box used in algorithms solving quantifier elimination problems (see [7]).

Algorithms computing a Cylindrical Algebraic Decomposition (see [10]) allow us to produce one point in each connected component of $\mathcal{S}_{+}, \mathcal{S}_{-}$or $\mathcal{S}$. Nevertheless the complexity of such algorithms is doubly exponential in the number of variables and their implementations are limited to problems having 3 or 4 variables.

Algorithms based on the critical point method are provided in [21, 22, 23, 33, 5, 6]. The classical strategy is to exhibit a hypersurface such that each connected component of $\mathcal{S}_{+}$ (resp. $\mathcal{S}_{-}$or $\mathcal{S}$ ) contains a connected component of the real counterpart of the exhibited hypersurface. Indeed, given an infinitesimal $\varepsilon$, denote by $\mathcal{H}_{\varepsilon} \subset \mathbb{C}\langle\varepsilon\rangle^{n}$ the hypersurface defined by $f-\varepsilon=0$. By the mean value theorem, each connected component of the embedding of $\mathcal{S}_{+}$in $\mathbb{R}\langle\varepsilon\rangle^{n}$ contains a connected component of $\mathcal{H}_{\varepsilon} \cap \mathbb{R}\langle\varepsilon\rangle^{n}$. Hence, the problem is reduced to compute at least one point in each connected component of the real counterpart of the hypersurface $\mathcal{H}_{\varepsilon}$.

Computing at least one point in each connected component of a real hypersurface. Consider a hypersurface $\mathcal{H} \subset \mathbb{C}^{n}$. We focus now on the state of the art on algorithms computing at least one point in each connected component (i.e. sampling points) of $\mathcal{H} \cap \mathbb{R}^{n}$. This problem is tackled by the critical point method. Its principle is the following: choose a polynomial mapping $\phi: \mathcal{H} \cap \mathbb{R}^{n} \rightarrow \mathbb{R}$ reaching its extrema in each connected component of $\mathcal{H} \cap \mathbb{R}^{n}$ and such that its critical locus is zero-dimensional or empty. When $\mathcal{H}$ is smooth, $\phi$ can be the square of the euclidean distance to a generically chosen point of $\mathbb{Q}^{n}$. When, additionally, $\mathcal{H} \cap \mathbb{R}^{n}$ is known to be compact, $\phi$ can be the projection on a line.

In [5], computing sampling points in $\mathcal{H} \cap \mathbb{R}^{n}$ is reduced to computing sampling points of a smooth hypersurface whose real counterpart is compact by introducing several infinitesimals. Thus, projection functions are used. The algorithms are deterministic and their complexity is $(2 D)^{\mathcal{O}(n)}$ arithmetic operations in $\mathbb{Q}$. Algebraic manipulations are performed to avoid a computation of Gröbner bases and lead to encode critical points as solutions of a zerodimensional polynomial system generating an ideal having always a degree $2 D(2 D-1)^{n}$. Moreover, all the computations are performed over a Puiseux series field. Thus, there is no hope to obtain an efficient practical behaviour of these algorithms.

In [36, 1, 4, 3, the authors use the square of the euclidean distance to a generically chosen point $A$ in $\mathbb{Q}^{n}$. Algorithms dealing with the case where $\mathcal{H}$ is not smooth are provided in [36. 1]. The one of [36] uses infinitesimal deformations. The one of [1] processes by performing 
a recursive study of the singular locus until it has dimension 0 or is empty. Because of the choice of $A$, the deterministic complexity of the algorithm of [36] is $D^{\mathcal{O}\left(n^{2}\right)}$. Nevertheless, in practice, the first choice is suitable to obtain zero-dimensional critical loci, so that under this assumption, which is satisfied in practice, the complexity of [36] is $D^{\mathcal{O}(n)}$. The complexity of [1] is not well-controlled even if in singular situations it behaves better than the ones based on infinitesimal deformations. The algorithms of [4, 3] use the geometric resolution algorithm which is probabilistic. Their complexity is polynomial in $n$, the evaluation complexity of the input polynomial and an intrinsic geometric degree $\delta$ which is dominated by $D^{n}$.

In the smooth case, these contributions are improved in [39]: generic projection functions are used even in non-compact situations instead of distance functions to a generic point. The genericity of the choice of projection functions is necessary to ensure properness properties. As in the case of algorithms using distance functions, in practice, the first choices are suitable. Using elimination algorithms based on the geometric resolution, this leads to a probabilistic algorithm whose arithmetic complexity is polynomial in $n$, the evaluation complexity of the input polynomial, and an intrinsic geometric degree $\delta$ which is dominated by $D(D-1)^{n-1}$. One can also use Gröbner bases. Making the assumptions that the first choice of projections is suitable, the complexity becomes $D^{\mathcal{O}(n)}$. This work is generalized to the case of singular hypersurfaces in [41]. The algorithms relying on [39] are the most efficient in practice and are implemented in 41 .

The output of all these algorithms are critical points encoded by a rational parameterization:

$$
\left\{\begin{array}{ccc}
X_{n} & = & \frac{q_{n}(T)}{q_{0}(T)} \\
& \vdots \\
X_{1} & = & \frac{q_{1}(T)}{q_{0}(T)} \\
q(T) & = & 0
\end{array}\right.
$$

where $T$ is a new variable, and $q, q_{0}, q_{1}, \ldots, q_{n}$ are univariate polynomials in $\mathbb{Q}[t]$. Such a rational parametrization can be obtained either by linear algebra computations in a quotientalgebra (see [35]) or directly by the geometric resolution algorithm (see [19, 17, 18, 20, 31]).

As recalled above, the classical strategy to compute at least one point in each connected component implies to apply the aforementioned algorithms in the case of a hypersurface defined by a polynomial with coefficients in $\mathbb{Q}(\varepsilon)$. Thus, the output is a rational parameterization with coefficients in $\mathbb{Q}(\varepsilon)$. Once it is obtained, a small enough specialization for $\varepsilon$ is obtained by computing the discriminant of $q$ with respect to $T$ and choosing a specialization less than the smallest absolute value of the real roots of this discriminant. Thus, the final output is smaller than the rational parameterization with coefficients in $\mathbb{Q}(\varepsilon)$. Moreover, computing rational parameterizations with coefficients in $\mathbb{Q}(\varepsilon)$ is hard in practice: infinitesimal arithmetics spoil the practical behaviour of elimination algorithms due to problems appearing in memory management and the over-cost of arithmetic operations. 
Substituting infinitesimal deformations by a pre-computation of generalized critical values. Remark that in order to obtain one point in each connected component in $\mathcal{S}_{+}$ (resp. $\mathcal{S}_{-}$or $\mathcal{S}$ ), it is sufficient to substitute a priori the infinitesimal $\varepsilon$ appearing in $f-\varepsilon$ by a small enough positive rational number $e \in \mathbb{Q}$. The problem is to ensure that the chosen rational number is small enough which means here that for each connected component $S$ of $\mathcal{S}_{+}$, there exists a connected component of the real counter part of the hypersurface defined by $f-e=0$ which is contained in $S$. This can be done by determining $e_{0} \in \mathbb{R}$ such that for all $e \in] 0, e_{0}[$, there exists a diffeomorphism $\varphi$ such that the following diagram commutes:

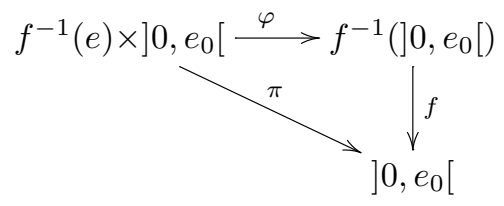

where $\pi$ is the canonical projection on the second member of the cartesian product $\left.f^{-1}(e) \times\right] 0, e_{0}[$.

Such a topological property is obtained by ensuring that the interval $I=] 0, e_{0}$ [ has an

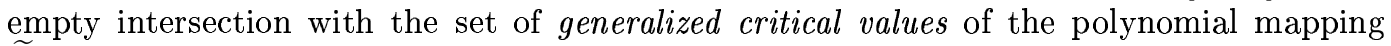
$\widetilde{f}: x \in \mathbb{R}^{n} \rightarrow f(x) \in \mathbb{R}$. This set of generalized critical values is denoted by $K(f)$ in the sequel. This set is defined and studied in [32]. A real number $c \in \mathbb{R}$ is a generalized critical value of a mapping $\widetilde{f}$ if and only if it is either a critical value of $\widetilde{f}$ or there exists a sequence of points $\left(z_{\ell}\right)_{\ell \in \mathbb{N}}$ such that $f\left(z_{\ell}\right)$ tends to $c$ when $\ell$ tends to $\infty,\left\|z_{\ell}\right\|$ tends to $\infty$ when $\ell$ tends to $\infty$ and $\left\|z_{\ell}\right\| .\left\|d_{z_{\ell}} f\right\|$ tends to 0 when $\ell$ tends to $\infty$. In the latter case, $c$ is said to be an asymptotic critical value. Degree bounds are provided in [26]. An algorithm computing them is described in [32]. This algorithm works as follows: denoting by $I$ the ideal

$$
I=\left\langle f-T,\left(\frac{\partial f}{\partial X_{i}}-a_{i}\right)_{i \in\{1, \ldots, n\}},\left(X_{i} \frac{\partial f}{\partial X_{j}}-a_{i, j}\right)_{(i, j) \in\{1, \ldots, n\}^{2}}\right\rangle
$$

where $a_{1}, \ldots, a_{1,1}, \ldots, a_{n, n}$ and $T$ are new variables, compute

$$
J=I \cap \mathbb{Q}\left[T, a_{1}, \ldots, a_{n}, a_{1,1}, \ldots, a_{n, n}\right] .
$$

Generalized critical values are solutions of

$$
J+\left\langle a_{1}, \ldots, a_{n}, a_{11}, a_{n, n}\right\rangle .
$$

Thus, this algorithm requires to perform algebraic elimination of variables on the ideal $I$ defined with polynomials involving $n^{2}+2 n+1$ variables. Moreover, the degree of $I$ can equal $D^{n}$ (where $D$ is the degree of $f$ ). Obviously, its practical behaviour is inefficient.

We provide here an algorithm computing efficiently the set of generalized critical values of a polynomial mapping from $\mathbb{R}^{n}$ to $\mathbb{R}$. A probabilistic version of this algorithm has a complexity within $D^{\mathcal{O}(n)}$ arithmetic operations in $\mathbb{Q}$ which is polynomial in the size of the output in worst-cases. 
This allows us to substitute the use of infinitesimal deformations by a pre-computation of generalized critical values in order to compute at least one point in each connected component of a semi-algebraic set defined by a single inequality. The algorithm we obtain is efficient in practice and its probabilistic versions have a complexity within $\mathcal{O}\left(n^{7} D^{4 n}\right)$ arithmetic operations in $\mathbb{Q}$. We also show how to apply our contribution to the problem of deciding if a hypersurface contains real regular points. Our algorithmic contributions have been implemented and we describe at the end of the paper how they have been applied on concrete applications which are unreachable with anterior methods.

Plan of the paper. The paper is organized as follows. In Section 2, we recall the definition and basic properties of generalized critical values which can be found in [32. In Section 3] we provide geometric results which, up to a generic linear change of the variables $X_{1}, \ldots, X_{n}$, characterize generalized critical values as the set of non-properness of a projection on a line restricted to a 1-dimensional polar variety. In Section 4 we show how to obtain a first algorithm computing generalized critical values which is directly based on the geometric results of Section 3 Then, we prove that these computations reduce to computing classical critical values on the one hand, and critical values at infinity on the other hand. In Section [5 we describe an algorithm computing at least one point in each connected component of a semi-algebraic set defined by a single inequality, which is based on the computation of generalized critical values. In Section [6] we show how to apply our contributions to determining if a hypersurface contains real regular points. Finally, Section 7 contains some benchmarks illustrating the practical efficiency of our algorithms and showing these methods are already promising to deal with problems having more than 4 variables.

Acknowledgments. The author thanks É. Schost and P. Trébuchet for fruitful discussions and comments about this work.

\section{Definition and first properties of generalized critical values}

In this section, we recall the definitions and basic properties of generalized critical values which can be found in [32].

Definition 1 A complex number $c \in \mathbb{C}$ is a critical value of the mapping $f: y \in \mathbb{C}^{n} \rightarrow f(y)$ if and only if there exists $z \in \mathbb{C}^{n}$ such that $f(z)=c$ and $\frac{\partial f}{\partial X_{1}}(z)=\cdots=\frac{\partial f}{\partial X_{n}}(z)=0$.

$A$ complex number $c \in \mathbb{C}$ is an asymptotic critical value of the mapping $f: y \in \mathbb{C}^{n} \rightarrow f(y)$ if and only if there exists a sequence of points $\left(z_{\ell}\right)_{\ell \in \mathbb{N}} \subset \mathbb{C}^{n}$ such that:

- $f\left(z_{\ell}\right)$ tends to $c$ when $\ell$ tends to $\infty$.

- $\left\|z_{\ell}\right\|$ tends to $+\infty$ when $\ell$ tends to $\infty$. 
- for all $(i, j) \in\{1, \ldots, n\}\left\|X_{i}\left(z_{\ell}\right)\right\| \cdot\left\|\frac{\partial f}{\partial X_{j}}\left(z_{\ell}\right)\right\|$ tends to 0 when $\ell$ tends to $\infty$.

Remark 1 Remark that any statement of the following kind: given a polynomial mapping $\varphi: \mathbb{R}^{n} \rightarrow \mathbb{R}^{q}$ and a point $y \in \mathbb{R}^{q}$, there exists a sequence of points $\left(z_{\ell}\right)_{\ell}$ lying in a semialgebraic set $\mathcal{S} \subset \mathbb{R}^{n}$ and a point $y \in \mathbb{R}^{q}$ such that:

- $\left\|z_{\ell}\right\|$ tends to $\infty$ when $\ell$ tends to $\infty$;

- $\varphi\left(y_{\ell}\right)$ tends to $y$ when $\ell$ tends to $\infty$;

can be rephrased using a quantified first order formula $\Phi$ over the reals. Then, from Tarski-Seidenberg's Theorem, the set of points satisfying $\Phi$ is a semi-algebraic set whose Zariski-closure is not zero-dimensional (since $\left\|z_{\ell}\right\|$ is supposed to tend to $\infty$ ). Thus, using the curve selection Lemma, the sequence of points in the above statement can be substituted by the existence of a semi-algebraic curve $\gamma:] 0,1\left[\rightarrow \mathbb{R}^{n}\right.$ such that $\|\gamma(t)\|$ tends to $\infty$ when $t \rightarrow 1$ and $\varphi(\gamma(t))$ tends to $y$ when $t \rightarrow 1$.

Example 1 Consider the following polynomial in $Q\left[X_{1}, X_{2}\right]$

$$
f=X_{1}\left(X_{1} X_{2}-1\right)
$$

and the mapping $\widetilde{f}:\left(x_{1}, x_{2}\right) \rightarrow f\left(x_{1}, x_{2}\right)$. This mapping has obviously no critical value since $\left\langle f-T, \frac{\partial f}{\partial X_{1}}, \frac{\partial f}{\partial X_{2}}\right\rangle=\mathbb{Q}\left[X_{1}, X_{2}, T\right]$. Suppose now that there exists a sequence of points $z_{\ell}$ such that:

- $\left\|z_{\ell}\right\|$ tends to $+\infty$ when $\ell$ tends to $\infty$.

- for all $(i, j) \in\{1,2\}\left\|X_{i}\left(z_{\ell}\right)\right\| \cdot\left\|\frac{\partial f}{\partial X_{j}}\left(z_{\ell}\right)\right\|$ tends to 0 when $\ell$ tends to $\infty$.

This implies that $X_{1}^{2}\left(z_{\ell}\right)$ tends to 0 when $\ell$ tends to $\infty$, which implies that $X_{1}\left(z_{\ell}\right)$ tends to 0 when $\ell$ tends to $\infty$, and $X_{2} X_{1}^{2}\left(z_{\ell}\right)$ tends to 0 when $\ell$ tends to $\infty$. Finally, $f\left(z_{\ell}\right)$ tends to 0 when $\ell$ tends to $\infty$. Thus, 0 is an asymptotic critical value of the mapping $\widetilde{f}$. We will see further that it is the only one.

Consider now the following example in 3 variables:

$$
f=X_{1}+X_{1}^{2} X_{2}+X_{1}^{4} X_{2} X_{3}
$$

In [32], the authors prove that the set of generalized critical values of the mapping sending $x \in \mathbb{C}^{n}$ to $f(x)$ is $\{0\}$ by using a similar reasoning as the above.

In [32], the authors prove the following result which can be seen as a generalized Sard's theorem for generalized critical values.

Theorem 1 Let $f$ be a polynomial in $\mathbb{Q}\left[X_{1}, \ldots, X_{n}\right]$ of degree D. The set of generalized critical values $K(f)$ of the mapping $\tilde{f}: x \in \mathbb{C}^{n} \rightarrow f(x) \in \mathbb{C}$ is Zariski-closed in $\mathbb{C}$.

Moreover, $D \sharp K_{\infty}(f)+\sharp K_{0}(f) \leq D^{n}-1$ 
Given $f \in \mathbb{Q}\left[X_{1}, \ldots, X_{n}\right]$, consider a mapping $f_{\mathbb{C}}: x \in \mathbb{C}^{n} \rightarrow f(x) \in \mathbb{C}$ and an open subset $F_{\mathbb{C}}$ of $\mathbb{C}$. We say that $f_{\mathbb{C}}$ realizes a locally trivial fibration on $\mathbb{C}^{n} \backslash f_{\mathbb{C}}^{-1}\left(F_{C}\right)$ if for all connected open set (for the euclidean topology) $U_{\mathbb{C}} \subset \mathbb{C} \backslash F_{\mathbb{C}}$, for all $e \in U_{\mathbb{C}}$ denoting by $\pi_{\mathbb{C}}$ the projection on the second member of the cartesian product $f_{\mathbb{C}}^{-1}(e) \times U_{\mathbb{C}}$, the following diagram

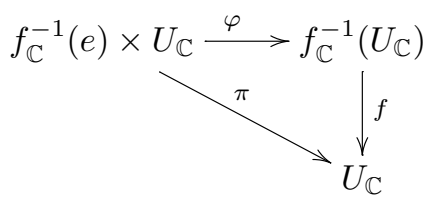

The above definition is also used for polynomial mappings from $\mathbb{R}^{n}$ to $\mathbb{R}$. Consider a mapping $f_{\mathbb{R}}: \mathbb{R}^{n} \rightarrow \mathbb{R}$ and an open subset $F_{\mathbb{R}}$ of $\mathbb{R}$. We say that $f_{\mathbb{R}}$ realizes a locally trivial fibration on $\mathbb{R}^{n} \backslash f_{\mathbb{R}}^{-1}\left(F_{C}\right)$ if for all connected open set (for the euclidean topology) $U_{\mathbb{R}} \subset \mathbb{C} \backslash F_{\mathbb{R}}$, for all $e \in U_{\mathbb{R}}$ denoting by $\pi_{\mathbb{R}}$ the projection on the second member of the cartesian product $f_{\mathbb{R}}^{-1}(e) \times U_{\mathbb{R}}$, the following diagram

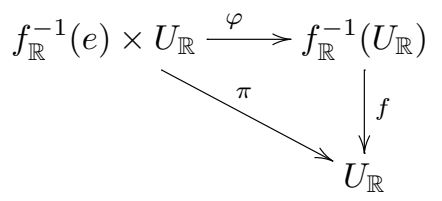

is commutative.

The main interest of the set generalized critical values relies on its topological properties which are summarized below and proved in [32].

Theorem 2 The mapping $f_{\mathbb{C}}$ realizes a locally trivial fibration in $\mathbb{C}^{n} \backslash f_{\mathbb{C}}^{-1}\left(K\left(f_{\mathbb{C}}\right)\right)$. The mapping $f_{\mathbb{R}}$ realizes a locally trivial fibration in $\mathbb{R}^{n} \backslash f_{\mathbb{R}}^{-1}\left(K\left(f_{\mathbb{R}}\right)\right)$.

Example 2 Consider the examples given above. We have proved that for both examples 0 is an asymptotic critical value. Remark that the fiber of both considered mappings above 0 is reducible while a generic fiber is irreducible. This is characteristic to a change of topology and is easily visualized on Figure 1 illustrating the example $f=X_{1}\left(X_{1} X_{2}-1\right)$.

Nevertheless, note that a mapping can realize a locally trivial fibration even if there exists a generalized critical value in I. To illustrate this fact, consider the following example:

$$
f=-X_{2}\left(2 X_{1}^{2} X_{2}^{2}-9 X_{1} X_{2}+12\right)
$$

which realizes a locally trivial fibration around 0 as shown in Figure 0 but is such that $K(f)=\{0\}$. 


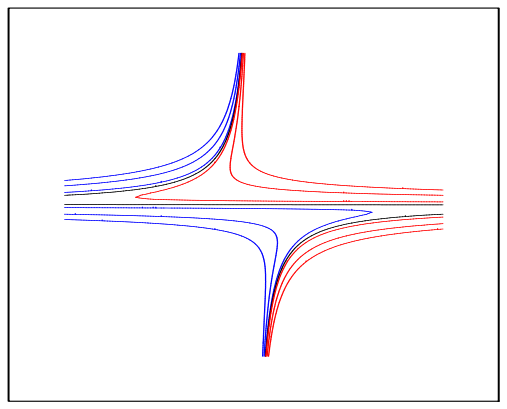

Figure 1: Existence of generalized critical values and change in topology

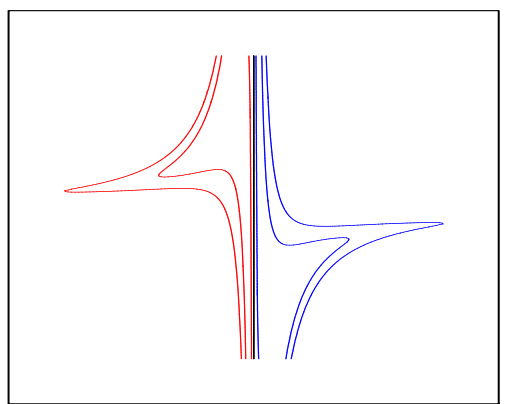

Figure 2: Existence of generalized critical values and no change in topology

$\mathrm{RR} \mathrm{n}^{\circ} 5995$ 
Thus, $K(f)$ is Zariski-closed, degree bounds on $K(f)$ are Bézout-like degree bounds and its topological properties ensure that there is no topological change in the fibers of $f$ taken above any interval of $\mathbb{R}$ which has an empty intersection with $K(f)$.

Denote by $G L_{n}(\mathbb{C})$ the set of $n$-square invertible matrices with coefficients in $\mathbb{C}$. Consider now $\mathbf{A} \in G L_{n}(\mathbb{C})$ and denote by $f^{\mathbf{A}}$ the polynomial $f(\mathbf{A X})$ where $\mathbf{X}$ denotes $\left(X_{1}, \ldots, X_{n}\right)$. Moreover, given $\left\{f_{1}, \ldots, f_{s}\right\}$ in $\mathbb{Q}\left[X_{1}, \ldots, X_{n}\right]$ and an algebraic variety $\mathcal{V} \subset \mathbb{C}^{n}$ defined by $f_{1}=\cdots=f_{s}=0$, we denote by $\mathcal{V}^{\mathbf{A}}$ the algebraic variety defined by $f_{1}^{\mathbf{A}}=\cdots=f_{s}^{\mathbf{A}}=0$.

The following lemma is an immediate consequence of Definition 1 and will be used in the sequel.

Lemma 1 For all $\mathbf{A} \in G L_{n}(\mathbb{Q}), K(f)$ equals $K\left(f^{\mathbf{A}}\right), K_{0}(f)$ equals $K_{0}\left(f^{\mathbf{A}}\right)$ and $K_{\infty}(f)$ equals $K_{\infty}\left(f^{\mathbf{A}}\right)$.

If $c$ is a critical value (resp. an asymptotic critical value) of $f$, then for all $e \in \mathbb{Q}, c-e$ is a critical value (resp. an asymptotic critical value) of $f+e$.

Using Remark 1, the following lemma is also immediate and is used further.

Lemma 2 Let $f$ be a polynomial in $\mathbb{Q}\left[X_{1}, \ldots, X_{n}\right]$. Consider $c \in \mathbb{C}$ and $\left(z_{\ell}\right)_{\ell \in \mathbb{N}} \subset \mathbb{C}^{n}$ be a sequence of points such that:

- $f\left(z_{\ell}\right)$ tends to $c$ when $\ell$ tends to $\infty$;

- $\left\|z_{\ell}\right\|$ tends to $\infty$ when $\ell$ tends to $\infty$;

- $\left\|z_{\ell}\right\| . \mid\left\|d_{z_{\ell}} f\right\|$ tends to 0 when $\ell$ tends to $\infty$.

Denote by $\mathrm{X}$ the vector $X_{1}, \ldots, X_{n}$. There exists a Zariski-closed subset $\mathcal{A} \subsetneq G L_{n}(\mathbb{C})$ such that for all $\mathbf{A} \in G L_{n}(\mathbb{Q}) \backslash \mathcal{A},\left\|\mathbf{A X}\left(z_{\ell}\right)\right\|$ tends to $\infty$ when $\ell$ tends to $\infty$.

In the sequel, for the sake of simplicity, we identify a polynomial $f \in \mathbb{Q}\left[X_{1}, \ldots, X_{n}\right]$ with the mapping $f_{\mathbb{C}}: x \in \mathbb{C}^{n} \rightarrow f(x) \in \mathbb{C}$.

\section{Geometric results}

Let $f$ be a polynomial in $\mathbb{Q}\left[X_{1}, \ldots, X_{n}\right], \mathcal{H} \subset \mathbb{C}^{n+1}$ be the hypersurface defined by $f-T=0$ (where $T$ is a new variable). Given $x=\left(x_{1}, \ldots, x_{n}\right) \in \mathbb{C}^{n}$, we denote by $F_{i}: \mathbb{C}^{n} \rightarrow \mathbb{C}^{n+1}$ the polynomial mapping sending $x$ to:

$$
\left(\left(\partial f / \partial X_{i}\right)(x),\left(X_{1} \partial f / \partial X_{i}\right)(x), \ldots,\left(X_{n} \partial f / \partial X_{i}\right)(x)\right)
$$

and by $\widetilde{F}_{i}: \mathbb{C}^{n} \rightarrow \mathbb{C}^{i n+i+1}$ the polynomial mapping sending $x$ to:

$$
\left(F_{1}(x), F_{2}(x), \ldots, F_{i}(x), f(x)\right) .
$$


We consider in the sequel the polynomial mapping $\phi: \mathbb{C}^{n} \rightarrow \mathbb{C}^{n^{2}+n+1}$ sending $x=$ $\left(x_{1}, \ldots, x_{n}\right)$ to

$$
\left(F_{1}(x), \ldots, F_{n}(x), f(x)\right)
$$

which coincides with $\widetilde{F}_{n}$. For any polynomial mapping $\psi$, we denote by $\Gamma_{\psi}$ the image of $\psi$ and by $\bar{\Gamma}_{\psi}$ its Zariski-closure. For $(i, j) \in\{1, \ldots, n\}^{2}$, we introduce new variables $a_{i}$, and $a_{i, j}$ such that $\bar{\Gamma}_{\phi}$ is defined by a set of generators of the ideal:

$$
\left\langle f-T,\left(\partial f / \partial X_{i}-a_{i}\right)_{i \in\{1, \ldots, n\}},\left(X_{i} . \partial f / \partial X_{j}-a_{i, j}\right)_{(i, j) \in\{1, \ldots, n\}^{2}}\right\rangle
$$

intersected with the polynomial ring $\mathbb{Q}\left[T, a_{1}, \ldots, a_{n}, a_{1,1}, \ldots, a_{n, n}\right]$.

Let $L_{i} \subset \mathbb{C}^{i n+i+1}$ be the coordinate axis of $T$, i.e. the line defined by:

$$
a_{1}=\cdots=a_{i}=a_{1,1}=\cdots=a_{n, 1}=\cdots=a_{1, i}=\cdots=a_{n, i}=0 .
$$

The line $L_{n}$ is denoted by $L$ in the sequel.

Kurdyka and its collaborators prove that $\bar{\Gamma}_{\phi} \cap L$ equals the set of generalized critical values of $f$ (see [32, 26]. The set of asymptotic critical values of $f$, denoted by $K_{\infty}(f)$, is characterized as the intersection of the set of non-properness of $\phi$ with $L$.

\subsection{Geometric characterization of generalized critical values under properness assumptions}

In the sequel, for $i=n, \ldots, 2$, we consider projections:

$$
\begin{array}{rlcc}
\Pi_{i}: & \mathbb{C}^{n+1} & \rightarrow & \mathbb{C}^{i} \\
\left(x_{1}, \ldots, x_{n}, t\right) & \mapsto & \left(x_{n-i+2}, \ldots, x_{n}, t\right)
\end{array}
$$

For $i=1, \ldots, n-1$, let $W_{n-i} \subset \mathbb{C}^{n+1}$ denotes the Zariski-closure of the constructible set defined by:

$$
f-T=\frac{\partial f}{\partial X_{1}}=\cdots=\frac{\partial f}{\partial X_{i}}=0, \quad \frac{\partial f}{\partial X_{i+1}} \neq 0 .
$$

For simplicity, $W_{n}$ denotes $\mathcal{H}$.

In the sequel, we consider maps between complex or real algebraic varieties. The notion of properness of such maps will be relative to the topologies induced by the metric topologies of $\mathbb{C}$ or $\mathbb{R}$. A map $\phi: V \rightarrow W$ of topological spaces is said to be proper at $w \in W$ if there exists a neighborhood $B$ of $w$ such that $f^{-1}(\bar{B})$ is compact (where $\bar{B}$ denotes the closure of $B)$. The map $\phi$ is said to be proper if it is proper at all $w \in W$.

Given $\mathbf{A} \in G L_{n}(\mathbb{Q})$ and $j \in\{2, \ldots, n\}$, we say that the property $\mathcal{P}_{j}(\mathbf{A})$ is satisfied if and only if for all $i \in\{j, \ldots, n\}$, the mapping $\Pi_{i}$ restricted to $W_{i}^{\mathbf{A}}$ is proper and the restriction of the map $\Pi_{i+1}$ to $W_{i}$ is birational onto its image.

In the sequel, we suppose that there exists a Zariski-closed subset $\mathcal{A} \subsetneq G L_{n}(\mathbb{Q})$ such that for all $\mathbf{A} \in G L_{n}(\mathbb{Q}) \backslash \mathcal{A}$ and $j \in\{2, \ldots, n\}$, the property $\mathcal{P}_{j}(\mathbf{A})$ is satisfied. 
Remark 2 Remark that from the algebraic Bertini-Sard theorem [44], if $\mathcal{P}(\mathbf{A})$ is true, $\Pi_{i}$ restricted to $W_{i}$ is a finite map and then $W_{i}^{\mathbf{A}}$ has dimension $i$.

We prove below that if $\mathcal{P}_{2}(\mathbf{A})$ is satisfied, given $c \in K_{\infty}(f)$, there exists a sequence of points $\left(z_{\ell}\right)_{\ell \in \mathbb{N}}$ in $W_{1}^{\mathbf{A}}$ such that:

- $f\left(z_{\ell}\right)$ tends to $c$ when $\ell$ tends to $\infty$

- $\left\|z_{\ell}\right\|$ tends to $\infty$ when $\ell$ tends to $\infty$

- $\left\|z_{\ell}\right\| .\left\|d_{z_{\ell}} f\right\|$ tends to 0 when $\ell$ tends to $\infty$

so that the existence of asymptotic critical values can be read off in $W_{1}$ which has dimension 1.

Proposition 1 Consider $c \in K_{\infty}(f)$. There exists a Zariski-closed subset $\mathcal{A} \subsetneq G L_{n}(\mathbb{C})$ such that for all $\mathbf{A} \in G L_{n}(\mathbb{Q}) \backslash \mathcal{A}$, there exists a sequence of points $\left(z_{\ell}\right)_{\ell \in \mathbb{N}}$ such that:

- for all $\ell \in \mathbb{N}, z_{\ell} \in W_{n-1}^{\mathbf{A}}$;

- $f^{\mathbf{A}}\left(z_{\ell}\right) \rightarrow c$ while $\ell \rightarrow \infty$;

- $\left\|z_{\ell}\right\|$ tends to $\infty$ when $\ell$ tends to $\infty$;

- $\left\|z_{\ell}\right\| .\left\|d_{z_{\ell}} f^{\mathbf{A}}\right\| \rightarrow 0$ while $\ell \rightarrow \infty$.

Proof. For the sake of simplicity, suppose that $\mathcal{P}_{n}\left(\mathbf{I}_{n}\right)$ is satisfied.

Consider the mapping $\phi: \mathcal{H} \subset \mathbb{C}^{n+1} \rightarrow \mathbb{C}^{2 n+2}$ which associates to a point $x=$ $\left(x_{1}, \ldots, x_{n}, t\right) \in \mathcal{H}$ the point:

$$
\left(x_{2}, \ldots, x_{n}, t, \frac{\partial f}{\partial X_{1}}(x), x_{1} \frac{\partial f}{\partial X_{1}}(x), \ldots, x_{n} \frac{\partial f}{\partial X_{1}}(x), t \frac{\partial f}{\partial X_{1}}(x)\right) \in \mathbb{C}^{2 n+2}
$$

Denote by $\left(a_{2}, \ldots, a_{n}, a_{n+1}, a_{0,1}, a_{1,1}, \ldots, a_{n+1,1}\right)$ the coordinates of the target space of $\phi$. Since for $i=2, \ldots, n, X_{i}=a_{i}, T=a_{n+1}$ and $X_{1}=\frac{a_{1,1}}{a_{0,1}}, X_{1}, \ldots, X_{n}$ and $T$ can be expressed as rational functions of coordinates in the target space of $\phi$ and then, the map $\phi$ is birational onto its image. Moreover, the graph of $\phi$, denoted by $\Gamma_{\phi}$ is an irreducible algebraic variety of $\mathbb{C}^{3 n+3}$ of dimension $n$. Then, there exists a Zariski-closed subset $\mathcal{Z} \subsetneq \mathbb{C}^{2 n+2}$ of maximal dimension $n-1$, such that specializing $n$ coordinates outside $\mathcal{Z}$, determines a unique point in the pre-image of $\phi$.

In the sequel, given a point $\underline{\alpha}=\left(\alpha_{2}, \ldots, \alpha_{n}\right) \in \mathbb{C}^{n-1}$ (resp. a complex number $\theta \in$ $\mathbb{C})$, such that $(\underline{\alpha}, \beta) \notin \mathcal{Z}$, we denote by $y(\underline{\alpha}, \beta)$ the point in the image of $\phi$ obtained by specializing the first $(n-1)$ coordinates (corresponding to $\left.x_{2}, \ldots, x_{n}\right)$ to $\underline{\alpha}$ and the $n+2$-th coordinate (corresponding to $x_{1} \frac{\partial f}{\partial X_{1}}$ ). Since $\phi$ is birational and since $\underline{\alpha}$ and $\beta$ are chosen generically, one can define $x(\underline{\alpha}, \beta)$ as the unique pre-image of $y(\underline{\alpha}, \beta)$.

Consider $c \in K_{\infty}(f)$, then there exists a sequence of points $\left(z_{\ell}\right)_{\ell \in \mathbb{N}} \subset \mathbb{C}^{n}$ such that: 
- $f\left(z_{\ell}\right)$ tends to $c$ when $\ell$ tends to $\infty$

- $\left\|z_{\ell}\right\|$ tends to $\infty$ when $\ell$ tends to $\infty$.

- $\left\|z_{\ell}\right\| .\left\|d_{z_{\ell}} f\right\|$ tends to 0 when $\ell$ tends to $\infty$.

Consider the images by $\phi$ of the points $\left(z_{\ell}, f\left(z_{\ell}\right)\right)$ and their first $n-1$ coordinates $\alpha_{\ell}$ and their $n+2$-th coordinates $\theta_{\ell}$. Note that such a choice implies that $\theta_{\ell}$ tends to 0 when $\ell$ tends to $\infty$. Since $f\left(z_{\ell}\right), 1 /\left\|z_{\ell}\right\|$ and $\left\|z_{\ell}\right\| .\left\|d_{z_{\ell}} f\right\|$ are Cauchy sequences, the doubly-indexed sequence $\underline{\alpha}_{i}, \theta_{\ell}$ is such that:

- (a) $f\left(x\left(\underline{\alpha}_{i}, \theta_{\ell}\right)\right)$ tends to $c$ when $i$ and $\ell$ tend to $\infty$;

- (b) $\left\|d_{x\left(\underline{\alpha}_{i}, \theta_{\ell}\right)} f\right\|$ tends to 0 when $i$ and $\ell$ tend to $\infty$.

- (c) $\left\|x\left(\underline{\alpha}_{i}, \theta_{\ell}\right)\right\|$ tends to $\infty$ when $i$ and $\ell$ tend to $\infty$.

- (d) $\left\|x\left(\underline{\alpha}_{i}, \theta_{\ell}\right)\right\| .\left\|d_{x\left(\underline{\alpha}_{i}, \theta_{\ell}\right)} f\right\|$ tends to 0 when $i$ and $\ell$ tend to $\infty$.

Moreover, without loss of generality, by disturbing infinitesimally $\underline{\alpha}_{i}$ and $\theta_{\ell}$, one can suppose that:

- (e) for all $i \in \mathbb{N}, \underline{\alpha}_{i}$ is chosen outside the Zariski-closed subset defined as the Zariskiclosure of the projection of $W_{n-2}$ onto $X_{2}, \ldots, X_{n}$.

- (f) from Lemma 2 one can suppose that up to a generic linear change of coordinates, $X_{1}\left(\alpha_{i}, \theta_{\ell}\right)$ tends to $\infty$ when $i$ and $\ell$ tend to $\infty$.

Since the map $\phi$ is birational, there exists an $n$-variate rational fraction $Q$ such that $X_{1}(x(\underline{\alpha}, \theta))$ is obtained by evaluating this rational fraction at $\underline{\alpha}, \theta$. Then, for a fixed integer $i_{0}, X_{1}\left(x\left(\underline{\alpha}_{i_{0}}, \theta_{\ell}\right)\right)$ has either a finite limit or tends to $\infty$ when $\ell$ tends to $\infty$. In the sequel, we prove that in both cases, $y\left(\underline{\alpha}_{i_{0}}, \theta_{\ell}\right)$ tends to a point whose last $n+2$ coordinates are null when $\ell$ tends to $\infty$.

Suppose first that $X_{1}\left(x\left(\underline{\alpha}_{i_{0}}, \theta_{\ell}\right)\right)$ has a finite limit when $\ell$ tends to $\infty$. Up to a generic linear change of variables, due to property (f), one can suppose that $\left\|X_{1}\left(x\left(\underline{\alpha}_{i}, \theta_{\ell}\right)\right)\right\|$ tends to $\infty$ when $i$ and $\ell$ tend to $\infty$. Thus, one can choose $i_{0}$ large enough to ensure that, if $X_{1}\left(x\left(\underline{\alpha}_{i_{0}}, \theta_{\ell}\right)\right)$ has a finite limit when $\ell$ tends to $\infty$, this limit is not 0 . This implies that the $n+1$-th coordinate of $y\left(\underline{\alpha}_{i_{0}}, \theta_{\ell}\right)$ (which corresponds to $\frac{\partial f}{\partial X_{1}}\left(\underline{\alpha}_{i_{0}}, \theta_{\ell}\right)$ ) tends to zero when $\ell \rightarrow \infty$ since one has seen that $\left(X_{1} \frac{\partial f}{\partial X_{1}}\right)\left(x\left(\underline{\alpha}_{i_{0}}, \theta_{\ell}\right)\right)$ tends to 0 and $X_{1}\left(x\left(\underline{\alpha}_{i_{0}}, \theta_{\ell}\right)\right)$ is supposed to have a finite limit which is not null.

This also implies that for $j=n+3, \ldots, 2 n+1$, the $j$-th coordinate of $y\left(\underline{\alpha}_{i_{0}}, \theta_{\ell}\right)$ tend to 0 when $\ell \rightarrow \infty$ since these coordinates can be rewritten as the product of one coordinate of $\underline{\alpha}_{i_{0}}$ (which is fixed) and the $(n+1)$-th of $y\left(\underline{\alpha}_{i_{0}}, \theta_{\ell}\right)$ which tends to 0 when $\ell$ tends to $\infty$.

Moreover, $f\left(x\left(\underline{\alpha}_{i_{0}}, \theta_{\ell}\right)\right)$ remains bounded (since $X_{1}\left(x\left(\underline{\alpha}_{i_{0}}, \theta_{\ell}\right)\right)$ has a finite limit), and has consequently a finite limit. Finally, this allows us to conclude that the last coordinate of $y\left(\underline{\alpha}_{i_{0}}, \theta_{\ell}\right)$ (which corresponds to $\left.t \frac{\partial f}{\partial X_{1}}\left(x\left(\underline{\alpha}_{i_{0}}, \theta_{\ell}\right)\right)\right)$ tends to 0 when $\ell$ tends to $\infty$. Thus, 
in this case, one has proved that $y\left(\underline{\alpha}_{i_{0}}, \theta_{\ell}\right)$ tends to a point whose last $n+2$ coordinates are null.

Suppose now that $X_{1}\left(x\left(\underline{\alpha}_{i_{0}}, \theta_{\ell}\right)\right)$ tends to $\infty$ when $\ell$ tends to $\infty$. This immediately implies that the $n+1$-th coordinate and, for $j=n+3, \ldots, 2 n+1$, the $j$-th coordinates of $y\left(\underline{\alpha}_{i_{0}}, \theta_{\ell}\right)$ tend to 0 when $\ell$ tend to $\infty$. It remains to prove that the last coordinate of $y\left(\underline{\alpha}_{i_{0}}, \theta_{\ell}\right)$ tends to 0 when $\ell$ tends to $\infty$.

Since $X_{1}\left(x\left(\underline{\alpha}_{i_{0}}, \theta_{\ell}\right)\right)$ tends to $\infty$ when $\ell$ tends to $\infty$, and using Remark 1 from the curve selection Lemma at infinity (see [32, Lemma 3.3, page 9], this implies there exists a semi-algebraic arc $\gamma_{i_{0}}:\left[0,1\left[\rightarrow \mathbb{R}^{n}\right.\right.$ such that

$$
\left\|\gamma_{i_{0}}(\rho)\right\| \rightarrow \infty \quad \text { and } \quad\left\|\gamma_{i_{0}}(\rho)\right\| .\left\|\frac{\partial f}{\partial X_{1}}\left(\gamma_{i_{0}}(\rho)\right)\right\| \rightarrow 0
$$

when $\rho$ tends to 1 . From Lojasiewicz's inequality at infinity [9, 2.3.11, p. 63], this implies that there exists an integer $N \geq 1$ such that:

$$
\forall \rho \in\left[0,1\left[, \quad \| \frac{\partial f}{\partial X_{1}}\left(\gamma_{i_{0}}(\rho)\right)\right)\|\leq\| \gamma_{i_{0}}(\rho) \|^{-1-\frac{1}{N}}\right.
$$

Following the same reasoning as in [32, Lemma 3.4, page 9], one can re-parameterize $\gamma_{i_{0}}$ such that $\gamma_{i_{0}}$ becomes a semi-algebraic function from $\left[0,+\infty\left[\right.\right.$ to $\mathbb{R}^{n}$ and $\lim _{\rho \rightarrow 1}\left\|\dot{\gamma}_{i_{0}}(\rho)\right\|=1$. Thus, the following yields:

$$
\forall \rho \in\left[0,+\infty\left[, \quad\left\|\frac{\partial f}{\partial X_{1}}\left(\gamma_{i_{0}}(\rho)\right)\right\| \cdot\left\|\dot{\gamma}_{i_{0}}(\rho)\right\| \leq\left\|\gamma_{i_{0}}(\rho)\right\|^{-1-\frac{1}{N}} \cdot\left\|\dot{\gamma}_{i_{0}}(\rho)\right\|\right.\right.
$$

and there exists $B \in \mathbb{R}$ such that

$$
\int_{0}^{\infty}\left\|\gamma_{i_{0}}(\rho)\right\|^{-1-\frac{1}{N}}\|\cdot\| \dot{\gamma}_{i_{0}}(\rho) \| d \rho \leq B
$$

Since

$$
\int_{0}^{\infty}\left\|\frac{\partial f}{\partial X_{1}}\left(\gamma_{i_{0}}(\rho)\right)\right\| \cdot\left\|\dot{\gamma}_{i_{0}}(\rho)\right\| d \rho \geq\left\|\int_{0}^{\infty} \frac{\partial f}{\partial X_{1}}\left(\gamma_{i_{0}}(\rho)\right) \cdot \dot{\gamma}_{i_{0}}(\rho) d \rho\right\|
$$

one has finally

$$
\left\|\int_{0}^{\infty} \frac{\partial f}{\partial X_{1}}\left(\gamma_{i_{0}}(\rho)\right) \cdot \dot{\gamma}_{i_{0}}(\rho) d \rho\right\| \leq B
$$

This implies that $f\left(X_{1}, \underline{\alpha}_{i_{0}}\right)$ is bounded along $\gamma_{i_{0}}$. Hence we have proved that $y\left(\underline{\alpha}_{i_{0}}, \theta_{\ell}\right)$ tends to a point whose last $n+2$ coordinates are null.

Let $y_{i_{0}}=\left(\underline{\alpha}_{i_{0}}, c_{i_{0}}, 0, \ldots, 0\right)$ be the limit of $y\left(\underline{\alpha}_{i_{0}}, \theta_{\ell}\right)$ and let $p_{i_{0}} \in \mathbb{C}^{n}$ be $\left(\underline{\alpha}_{i_{0}}, c_{i_{0}}\right)$ and $p_{\ell} \in \mathbb{C}^{n}$ be the point whose coordinates are the $n$-first coordinates of $y\left(\underline{\alpha}_{i_{0}}, \theta_{\ell}\right)$. We prove now that $y_{i_{0}}$ belongs to the image of $\phi$.

Since the restriction to $\mathcal{H}$ of $\Pi_{n}$ is supposed to be proper, for all $\ell \in \mathbb{N}, \Pi_{n}^{-1}\left(p_{\ell}\right) \cap \mathcal{H} \neq \emptyset$ and there exists a ball centered at $p_{i_{0}}$ such that $\Pi_{n}^{-1}(\mathcal{B})$ is compact. Moreover, remark that $x\left(\underline{\alpha}_{i_{0}}, \theta_{\ell}\right)$ belongs to $\Pi_{n}^{-1}\left(p_{\ell}\right)$. 
Thus, one can extract a converging subsequence from $\left(x\left(\underline{\alpha}_{i_{0}}, \theta_{\ell}\right)\right)_{\ell \in \mathbb{N}}$ and let $x_{i_{0}}$ be the limit of the chosen converging subsequence. Note that we have proved above that the evaluation of $\frac{\partial f}{\partial X_{1}}$ at $x\left(\underline{\alpha}_{i_{0}}, \theta_{\ell}\right)$ tends to 0 when $\ell$ tends to $\infty$ which implies that $\frac{\partial f}{\partial X_{1}}$ vanishes at $x_{i_{0}}$. Moreover, from property (e), $\frac{\partial f}{\partial X_{2}}$ does not vanish at $x_{i_{0}}$. Hence $x_{i_{0}}$ belongs to $W_{n-1}$ and $\phi\left(x_{i_{0}}\right)=y_{i_{0}}$ which implies that $y_{i_{0}}$ belongs to the image of $\phi$. To end the proof, note that, from properties (a), (c) and (d), $\left(f\left(x_{i_{0}}\right)\right)_{i_{0} \in \mathbb{N}}\left(\operatorname{resp}\right.$. $\left(\left\|x_{i_{0}}\right\|\right)_{i_{0} \in \mathbb{N}}$ and $\left(\left\|d_{x_{i_{0}}} f\right\|\right)_{i_{0} \in \mathbb{N}}$ and $\left.\left(\left\|x_{i_{0}}\right\| . \| d_{x_{i_{0}}} f||\right)_{i_{0} \in \mathbb{N}}\right)$ has the same limit when $i_{0}$ tends to $\infty$ as $\left(f\left(x\left(\underline{\alpha}_{i}, \theta_{\ell}\right)\right)\right)_{(i, \ell) \in \mathbb{N} \times \mathbb{N}}$ (resp. $\left\|d_{x\left(\underline{\alpha}_{i}, \theta_{\ell}\right)} f\right\|$ and $\left\|x\left(\underline{\alpha}_{i}, \theta_{\ell}\right)\right\|$ and $\left.\left\|x\left(\underline{\alpha}_{i}, \theta_{\ell}\right)\right\| .\left\|d_{x\left(\underline{\alpha}_{i}, \theta_{\ell}\right)} f\right\|\right)$ when $i$ and $\ell$ tend to $\infty$.

The following result tells that under some assumptions on the properness of some projections and the dimension of a polar variety, generalized critical values can be read off in the polar variety $W_{1}$.

Proposition 2 Consider $c \in K_{\infty}(f)$. There exists a a Zariski-closed subset $\mathcal{A} \subsetneq G L_{n}(\mathbb{C})$ such that for all $A \in G L_{n}(\mathbb{Q}) \backslash \mathcal{A}$, there exists a sequence of points $\left(z_{\ell}\right)_{\ell \in \mathbb{N}}$ such that:

- for all $\ell \in \mathbb{N}, z_{\ell} \in W_{1}^{\mathbf{A}}$;

- $f^{\mathbf{A}}\left(z_{\ell}\right) \rightarrow c$ when $\ell \rightarrow \infty$;

- $\left\|z_{\ell}\right\|$ tends to $\infty$ when $\ell$ tends to $\infty$;

- $\left\|z_{\ell}\right\| .\left\|d_{z_{\ell}} f^{\mathbf{A}}\right\| \rightarrow 0$ when $\ell \rightarrow \infty$.

The proof of the above result uses the same techniques than the ones used in the proof of Proposition 1 .

Proof. Given an integer $j$ in $\{n, \ldots, 2\}$, we say that property $\mathfrak{P}_{j}$ is satisfied if and only if the following assertion is true: let $c \in K_{\infty}(f)$, if the property $\mathcal{P}_{j}\left(\mathbf{I}_{n}\right)$ is satisfied, then there exists a sequence of points $\left(z_{\ell}\right)_{\ell \in \mathbb{N}}$ such that:

- for all $\ell \in \mathbb{N}, z_{\ell} \in W_{j-1}$;

- $f\left(z_{\ell}\right) \rightarrow c$ when $\ell \rightarrow \infty$;

- $\left\|z_{\ell}\right\|$ tends to $\infty$ when $\ell$ tends to $\infty$;

- $\left\|z_{\ell}\right\| .\left\|d_{z_{\ell}} f\right\| \rightarrow 0$ when $\ell \rightarrow \infty$.

The case $j=n$ is already proved in Proposition 1.

Suppose now $\mathfrak{P}_{j+1}$ is true and $\mathcal{P}_{j}\left(\mathbf{I}_{n}\right)$ is satisfied. We show below that this implies $\mathfrak{P}_{j}$.

Since $\mathfrak{P}_{j+1}$ is supposed to be true and $\mathcal{P}_{j+1}\left(\mathbf{I}_{n}\right)$ holds, then there exists a sequence of points $\left(z_{\ell}\right)_{\ell \in \mathbb{N}}$ such that:

- for all $\ell \in \mathbb{N}, z_{\ell} \in W_{j}$;

- $f\left(z_{\ell}\right) \rightarrow c$ when $\ell \rightarrow \infty$; 
- $\left\|z_{\ell}\right\|$ tends to $\infty$ when $\ell$ tends to $\infty$;

- $\left\|z_{\ell}\right\| .\left\|d_{z_{\ell}} f\right\| \rightarrow 0$ when $\ell \rightarrow \infty$.

We prove below that one can choose such a sequence $\left(z_{\ell}\right)_{\ell \in \mathbb{N}}$ in $W_{j-1}$ by using similar arguments to the ones used in the proof of Proposition 10

Consider the mapping $\phi: W_{j} \subset \mathbb{C}^{n+1} \rightarrow \mathbb{C}^{2 j+2}$ which associates to a point $x=$ $\left(x_{1}, \ldots, x_{n}, t\right) \in W_{j}$ the point:

$$
\left(x_{n-j+2}, \ldots, x_{n}, t, \frac{\partial f}{\partial X_{n-j+1}}(x),\left(x_{n-j+r} \frac{\partial f}{\partial X_{n-j+1}}(x)\right)_{r=1, \ldots, j}, t \frac{\partial f}{\partial X_{n-j+1}}(x)\right)
$$

Denote by $\left(a_{n-j+2}, \ldots, a_{n}, a_{n+1}, a_{0, n-j+1}, a_{n-j+1, n-j+1}, \ldots, a_{n+1, n-j+1}\right)$ the coordinates of the target space of $\phi$. Since for $i=n-j+2, \ldots, n, X_{i}=a_{i}, X_{n-j+1}=\frac{a_{n-j+1, n-j+1}}{a_{0, n-j+1}}$ $T=a_{n+1}$, and, since $\mathcal{P}_{j+1}\left(\mathbf{I}_{n}\right)$ holds, for $i=1, \ldots, n-j+1, X_{i}$ can be expressed as a rational fraction lying in $\mathbb{Q}\left(X_{n-j+1}, \ldots, X_{n}\right), X_{1}, \ldots, X_{n}$ and $T$ can be expressed as rational functions of coordinates in the target space of $\phi$ and then the map $\phi$ is bi-rational onto its image. Moreover, since $W_{j}$ has dimension $j$, the graph of $\phi$, denoted by $\Gamma_{\phi}$ is an irreducible algebraic variety of $\mathbb{C}^{n+2 j+3}$ of dimension $j$. Then, there exists a Zariski-closeed subset $\mathcal{Z} \subsetneq \mathbb{C}^{2 j+2}$ of maximal dimension $j-1$, such that specializing $j$ coordinates outside $\mathcal{Z}$, in the target space of $\phi$ determines a unique point in the pre-image of $\phi$.

Given a point $\underline{\alpha}=\left(\alpha_{n-j+2}, \ldots, \alpha_{n}\right) \in \mathbb{C}^{j-1}$ (resp. a complex number $\theta \in \mathbb{C}$ ), such that $(\underline{\alpha}, \beta) \notin \mathcal{Z}$, we denote by $y(\underline{\alpha}, \beta)$ the point in the image of $\phi$ obtained by specializing the first $(j-1)$ coordinates (corresponding to $\left.x_{n-j+2}, \ldots, x_{n}\right)$ to $\underline{\alpha}$ and the $j+2$-th coordinate (corresponding to $x_{n-j+1} \frac{\partial f}{\partial X_{n-j+1}}$ ). Since $\phi$ is birational and since $\underline{\alpha}$ and $\beta$ are chosen generically, one can define $x(\underline{\alpha}, \beta) \in W_{j}$ as the unique pre-image of $y(\underline{\alpha}, \beta)$.

Consider $c \in K_{\infty}(f)$, then, as in the proof of Proposition 1 one can choose sequences $\underline{\alpha}_{i}$ and $\theta_{\ell}$ such that:

- (a) $f\left(x\left(\underline{\alpha}_{i}, \theta_{\ell}\right)\right)$ tends to $c$ when $i$ and $\ell$ tend to $\infty$;

- (b) $\left\|d_{x\left(\underline{\alpha}_{i}, \theta_{\ell}\right)} f\right\|$ tends to 0 when $i$ and $\ell$ tend to $\infty$.

- (c) $\left\|x\left(\underline{\alpha}_{i}, \theta_{\ell}\right)\right\|$ tends to $\infty$ when $i$ and $\ell$ tend to $\infty$.

- (d) $\left\|x\left(\underline{\alpha}_{i}, \theta_{\ell}\right)\right\| .\left\|d_{x\left(\underline{\alpha}_{i}, \theta_{\ell}\right)} f\right\|$ tends to 0 when $i$ and $\ell$ tend to $\infty$.

Note that such a choice implies that $\theta_{\ell}$ tends to 0 when $\ell$ tends to $\infty$. Moreover, without loss of generality, by disturbing infinitesimally $\underline{\alpha}_{i}$ and $\theta_{\ell}$, one can suppose that:

- (e) for all $i \in \mathbb{N}, \underline{\alpha}_{i}$ is chosen outside the Zariski-closed subset defined as the Zariskiclosure of the projection of $W_{j-2}$ onto $X_{n-j+2}, \ldots, X_{n}$.

- (f) from Lemma 2 one can suppose that up to a generic linear change of coordinates, $X_{n-j+1}\left(\alpha_{i}, \theta_{\ell}\right)$ tends to $\infty$ when $i$ and $\ell$ tend to $\infty$ 
Since the map $\phi$ is birational, there exists an $n$-variate rational fraction $Q$ such that $X_{n-j+1}(x(\underline{\alpha}, \theta))$ is obtained by evaluating this rational fraction at $\underline{\alpha}, \theta$. Then, for a fixed integer $i_{0}, X_{n-j+1}\left(x\left(\underline{\alpha}_{i_{0}}, \theta_{\ell}\right)\right)$ has either a finite limit or tends to $\infty$ when $\ell$ tends to $\infty$. In the sequel, we prove that in both cases, $y\left(\underline{\alpha}_{i_{0}}, \theta_{\ell}\right)$ tends to a point whose last $j+2$ coordinates are null.

Suppose first that $X_{n-j+1}\left(x\left(\underline{\alpha}_{i_{0}}, \theta_{\ell}\right)\right)$ has a finite limit when $\ell$ tends to $\infty$. Up to a generic linear change of variables, due to property (f), one can suppose that $\left\|X_{n-j+1}\left(x\left(\underline{\alpha}_{i}, \theta_{\ell}\right)\right)\right\|$ tends to $\infty$ when $i$ and $\ell$ tend to $\infty$. Thus, one can choose $i_{0}$ large enough to ensure that if $X_{n-j+1}\left(x\left(\underline{\alpha}_{i_{0}}, \theta_{\ell}\right)\right)$ has a finite limit when $\ell$ tends to $\infty$, this limit is not 0 . This implies that the $j+1$-th coordinate of $y\left(\underline{\alpha}_{i_{0}}, \theta_{\ell}\right)$ tends to zero when $\ell \rightarrow \infty$.

This also implies that for $k=j+3, \ldots, 2 j+1$, the $k$-th coordinate of $y\left(\underline{\alpha}_{i_{0}}, \theta_{\ell}\right)$ tend to 0 when $\ell \rightarrow \infty$ since these coordinates can be rewritten as the product of one coordinate of $\underline{\alpha}_{i_{0}}$ and the $(j+1)$-th of $y\left(\underline{\alpha}_{i_{0}}, \theta_{\ell}\right)$ which tends to 0 when $\ell$ tends to $\infty$.

Moreover, $f\left(x\left(\underline{\alpha}_{i_{0}}, \theta_{\ell}\right)\right)$ remains bounded (since $X_{n-j+1}\left(x\left(\underline{\alpha}_{i_{0}}, \theta_{\ell}\right)\right)$ has a finite limit and the pre-image of $\mathbb{C}^{2 j+2}$ by $\phi$ lies in $W_{j}$ ), and has consequently a finite limit. Finally, this allows us to conclude that the last coordinate of $y\left(\underline{\alpha}_{i_{0}}, \theta_{\ell}\right)$ tends to 0 when $\ell$ tends to $\infty$. Thus, in this case, one has proved that $y\left(\underline{\alpha}_{i_{0}}, \theta_{\ell}\right)$ tends to a point whose last $j+2$ coordinates are null.

Suppose now that $X_{n-j+1}\left(x\left(\underline{\alpha}_{i_{0}}, \theta_{\ell}\right)\right)$ tends to $\infty$ when $\ell$ tends to $\infty$. This immediately implies that the $j+1$-th coordinate and, for $k=j+3, \ldots, 2 j+1$, the $k$-th coordinates of $y\left(\underline{\alpha}_{i_{0}}, \theta_{\ell}\right)$ tend to 0 when $\ell$ tend to $\infty$. It remains to prove that the last coordinate of $y\left(\underline{\alpha}_{i_{0}}, \theta_{\ell}\right)$ tends to 0 when $\ell$ tends to $\infty$.

Since $X_{n-j+1}\left(x\left(\underline{\alpha}_{i_{0}}, \theta_{\ell}\right)\right)$ tends to $\infty$ when $\ell$ tends to $\infty$, from the curve selection Lemma at infinity (see [32, Lemma 3.3, page 9], this implies there exists a semi-algebraic arc $\gamma_{i_{0}}$ : $\left[0,1\left[\rightarrow \mathbb{R}^{n}\right.\right.$ such that $\gamma_{i_{0}}\left(\left[0,1[)\right.\right.$ is included in the intersection of $W_{j}$ and of the linear subspace defined by $X_{k}=X_{k}\left(\underline{\alpha}_{i_{0}}\right)$ for $k=n-j+2, \ldots, n$ and

$$
\left\|\gamma_{i_{0}}(\rho)\right\| \rightarrow \infty \quad \text { and } \quad\left\|X_{n-j+1}\left(\gamma_{i_{0}}(\rho)\right)\right\| \cdot\left\|\frac{\partial f}{\partial X_{n-j+1}}\left(\gamma_{i_{0}}(\rho)\right)\right\| \rightarrow 0
$$

when $\rho$ tends to 1 . From Lojasiewicz's inequality at infinity [9. 2.3.11, p. 63], this implies that there exists an integer $N \geq 1$ such that:

$$
\forall \rho \in\left[0,1\left[, \quad \| \frac{\partial f}{\partial X_{n-j+1}}\left(\gamma_{i_{0}}(\rho)\right)\right)\|\leq\| X_{n-j+1}\left(\gamma_{i_{0}}(\rho)\right) \|^{-1-\frac{1}{N}}\right.
$$

Following the same reasoning as in [32, Lemma 3.4, page 9], one can re-parameterize $\gamma_{i_{0}}$ such that $\gamma_{i_{0}}$ becomes a semi-algebraic function from $\left[0,+\infty\left[\right.\right.$ to $\mathbb{R}^{n}$ and $\lim _{\rho \rightarrow 1}\left\|\dot{\gamma}_{i_{0}}(\rho)\right\|=1$. Thus, the following yields:

$$
\forall p \in\left[0,+\infty\left[, \quad\left\|\frac{\partial f}{\partial X_{n-j+1}}\left(\gamma_{i_{0}}(\rho)\right)\right\| .\left\|\dot{\gamma}_{i_{0}}(\rho)\right\| \leq\left\|X_{n-j+1}\left(\gamma_{i_{0}}(\rho)\right)\right\|^{-1-\frac{1}{N}} .\left\|\dot{\gamma}_{i_{0}}(\rho)\right\|\right.\right.
$$

and there exists $B \in \mathbb{R}$ such that

$$
\int_{0}^{\infty}\left\|\gamma_{i_{0}}(\rho)\right\|^{-1-\frac{1}{N}} \cdot\left\|\dot{\gamma}_{i_{0}}(\rho)\right\| d \rho \leq B
$$


Since

$$
\int_{0}^{\infty}\left\|\gamma_{i_{0}}(\rho)\right\|^{-1-\frac{1}{N}} \cdot\left\|\dot{\gamma}_{i_{0}}(\rho)\right\| d \rho \geq \int_{0}^{\infty}\left\|X_{n-j+1}\left(\gamma_{i_{0}}(\rho)\right)\right\|^{-1-\frac{1}{N}} \cdot\left\|\dot{\gamma}_{i_{0}}(\rho)\right\| d \rho
$$

and

$$
\int_{0}^{\infty}\left\|\frac{\partial f}{\partial X_{n-j+1}}\left(\gamma_{i_{0}}(\rho)\right)\right\| \cdot\left\|\dot{\gamma}_{i_{0}}(\rho)\right\| d \rho \geq\left\|\int_{0}^{\infty} \frac{\partial f}{\partial X_{n-j+1}}\left(\gamma_{i_{0}}(\rho)\right) \cdot \dot{\gamma}_{i_{0}}(\rho) d \rho\right\|
$$

one has finally

$$
\left\|\int_{0}^{\infty} \frac{\partial f}{\partial X_{n-j+1}}\left(\gamma_{i_{0}}(\rho)\right) \cdot \dot{\gamma}_{i_{0}}(\rho) d \rho\right\| \leq B
$$

Thus, the restriction of $f$ is bounded along $\gamma_{i_{0}}$. Hence we have proved that $y\left(\underline{\alpha}_{i_{0}}, \theta_{\ell}\right)$ tends to a point whose last $n+2$ coordinates are null.

Let $y_{i_{0}}=\left(\underline{\alpha}_{i_{0}}, c_{i_{0}}, 0, \ldots, 0\right)$ be the limit of $y\left(\underline{\alpha}_{i_{0}}, \theta_{\ell}\right)$ and let $p_{i_{0}} \in \mathbb{C}^{n}$ be $\left(\underline{\alpha}_{i_{0}}, c_{i_{0}}\right)$ and $p_{\ell} \in \mathbb{C}^{n}$ be the point whose coordinates are the $n$-first coordinates of $y\left(\underline{\alpha}_{i_{0}}, \theta_{\ell}\right)$. We prove now that $y_{i_{0}}$ belongs to the image of $\phi$.

Since the restriction to $W_{j}$ of $\Pi_{j}$ is supposed to be proper, for all $\ell \in \mathbb{N}, \Pi_{j}^{-1}\left(p_{\ell}\right) \cap W_{j} \neq \emptyset$ and there exists a ball centered at $p_{i_{0}}$ such that $\Pi_{j}^{-1}(\mathcal{B})$ is compact. Moreover, remark that $x\left(\underline{\alpha}_{i_{0}}, \theta_{\ell}\right)$ belongs to $\Pi_{j}^{-1}\left(p_{\ell}\right)$.

Thus, one can extract a converging subsequence from $\left(x\left(\underline{\alpha}_{i_{0}}, \theta_{\ell}\right)\right)_{\ell \in \mathbb{N}}$ and let $x_{i_{0}}$ be the limit of the chosen converging subsequence. Note that we have proved above that the evaluation of $\frac{\partial f}{\partial X_{n-j+1}}$ at $x\left(\underline{\alpha}_{i_{0}}, \theta_{\ell}\right)$ tends to 0 when $\ell$ tends to $\infty$ which implies that $\frac{\partial f}{\partial X_{n-j+1}}$ vanishes at $x_{i_{0}}$. Moreover, from property (e), $\frac{\partial f}{\partial X_{n-j+2}}$ does not vanish at $x_{i_{0}}$. Hence $x_{i_{0}}$ belongs to $W_{j-1}$ and $\phi\left(x_{i_{0}}\right)=y_{i_{0}}$ which implies that $y_{i_{0}}$ belongs to the image of $\phi$. To end the proof, note that, from properties (a), (c) and (d), $\left(f\left(x_{i_{0}}\right)\right)_{i_{0} \in \mathbb{N}}\left(\operatorname{resp} .\left(\left\|x_{i_{0}}\right\|\right)_{i_{0} \in \mathbb{N}}\right.$ and $\left(\left\|d_{x_{i_{0}}} f\right\|\right)_{i_{0} \in \mathbb{N}}$ and $\left.\left(\left\|x_{i_{0}}\right\| \cdot\left\|d_{x_{i_{0}}} f\right\|\right)_{i_{0} \in \mathbb{N}}\right)$ has the same limit when $i_{0}$ tends to $\infty$ as $\left(f\left(x\left(\underline{\alpha}_{i}, \theta_{\ell}\right)\right)\right)_{(i, \ell) \in \mathbb{N} \times \mathbb{N}}\left(\operatorname{resp} .\left\|d_{x\left(\underline{\alpha}_{i}, \theta_{\ell}\right)} f\right\|\right.$ and $\left\|x\left(\underline{\alpha}_{i}, \theta_{\ell}\right)\right\|$ and $\left.\left\|x\left(\underline{\alpha}_{i}, \theta_{\ell}\right)\right\| .\left\|d_{x\left(\underline{\alpha}_{i}, \theta_{\ell}\right)} f\right\|\right)$ when $i$ and $\ell$ tend to $\infty$.

\subsection{Ensuring properness properties}

We prove now that there exists a Zariski-closed subset $\mathcal{A} \in G L_{n}-\mathbb{C}$ ) such that for all $\mathbf{A} \in$ $G L_{n}(\mathbb{Q}) \backslash \mathcal{A}$, the property $\mathcal{P}_{1}(\mathbf{A})$ holds, which is summarized in the following proposition.

Proposition 3 There exists a Zariski-closed subset $\mathcal{A} \subsetneq G L_{n}(\mathbb{C})$ such that for all $\mathbf{A} \in$ $G L_{n}(\mathbb{Q}) \backslash \mathcal{A}$ and for all $j \in\{1, \ldots, n-1\}$ :

- $\Pi_{j}$ restricted to $W_{j}$ is proper.

- the restriction of $\Pi_{j+1}$ to $W_{j}$ is bi-rational onto its image. 
In [39, the authors prove that given a hypersurface $\mathcal{H} \subset \mathbb{C}^{n+1}$, there exists a Zariskiclosed subset $\mathcal{A} \subsetneq G L_{n+1}(\mathbb{C})$ such that for $j \in\{1, \ldots, n-1\}$ and for all $\mathbf{A} \in G L_{n+1}(\mathbb{Q}) \backslash \mathcal{A}$, $\Pi_{j}$ restricted to $W_{j}^{\mathbf{A}}$ is proper and satisfies a Nœther normalization property.

This result can not be used as stated in [39, since we consider here the hypersurface defined by $f-T=0$ and allow only change of variables on $X_{1}, \ldots, X_{n}$. Nevertheless, the incremental intersection process, originate from [19, 18, 17, which is used in the proof of [39] allows us to state:

Proposition 4 For $i=1, \ldots, n$, denote by $\Delta_{i}^{\mathbf{A}}$ the ideals associated to the Zariski-closure of the constructible set defined by:

$$
\frac{\partial f^{\mathbf{A}}}{\partial X_{1}}=\cdots=\frac{\partial f^{\mathbf{A}}}{\partial X_{i}}=0, \quad \frac{\partial f^{\mathbf{A}}}{\partial X_{i+1}} \neq 0
$$

There exists a Zariski-closed subset $\mathcal{A} \subsetneq G L_{n}(\mathbb{C})$ such that:

- for all $i \in\{1, \ldots, n\}$ and for all prime $P_{i}^{\mathbf{A}}$ associated to $\Delta_{i}^{\mathbf{A}}$, the extension $\mathbb{C}\left[\mathbf{X}_{\geq i+1}\right] \rightarrow$ $\mathbb{C}[\mathbf{X}] / P_{i}^{\mathbf{A}}$ is integral, where $\mathbf{X}_{\geq i+1}$ denotes $X_{i+1}, \ldots, X_{n}$ and $\mathbf{X}$ denotes $X_{1}, \ldots, X_{n}$.

- for all $i \in\{2, \ldots, n-1\}$, the restriction of the projection $\pi_{i}:\left(x_{1}, \ldots, x_{n}\right) \rightarrow\left(x_{i}, \ldots, x_{n}\right) \in$ $\mathbb{C}^{n-i+1}$ to the algebraic variety defined by $\Delta_{i}^{\mathbf{A}}$ is birational onto its image.

Using mutatis mutandis the proof of [39, Proposition 3, Section 2.5], which is based on [24. Lemma 3.10] relating the properness of $\pi_{i}$ to the fact that the above extensions are integral yields the following result:

Lemma 3 Denote by $\pi_{i+1}$ the projection $\left(x_{1}, \ldots, x_{n}\right) \in \mathbb{C}^{n} \rightarrow\left(x_{i+1}, \ldots, x_{n}\right) \in \mathbb{C}^{n-i}$. There exists a Zariski-closed subset $\mathcal{A} \subsetneq G L_{n}(\mathbb{C})$ such that for all $\mathbf{A} \in G L_{n}(\mathbb{Q}) \backslash \mathcal{A}$ and for all $i \in\{1, \ldots, n\}, \pi_{i+1}$ restricted to the algebraic variety defined by $\Delta_{i}^{\mathbf{A}}$ is proper.

Now, we prove that if $\pi_{i}$ restricted to the algebraic variety associated to $\Delta_{i}^{\mathbf{A}}$ is proper, then $\Pi_{i}$ restricted to $W_{i}^{\mathbf{A}}$ is proper. Indeed, suppose there exists $(x, t) \in \mathbb{C}^{i-1} \times \mathbb{C}$ such that $\Pi_{i}$ restricted to $W_{i}^{\mathbf{A}}$ is not proper at $(x, t)$. This means that there exists a ball $B \times U \subset$ $\mathbb{C}^{i-1} \times \mathbb{C}$ containing $(x, t)$ such that $\Pi_{i}^{-1}(B) \cap W_{i}^{\mathbf{A}}$ is not compact. Remark that in that case, the only variables which can tend to infinity are $X_{1}, \ldots, X_{n-i}$ or $X_{n-i+1}$. This implies that the projection of $\Pi_{i}^{-1}(B) \cap W_{i}^{\mathbf{A}}$ onto $X_{1}, \ldots, X_{n}$ is not compact. Moreover, the projection of $\Pi_{i}^{-1}(B) \cap W_{i}^{\mathbf{A}}$ onto $X_{1}, \ldots, X_{n}$ is contained in the pre-image by $\pi_{i}$ of $B$ which contains $x$. Thus, the non-properness of $\Pi_{i}$ restricted to $W_{i}^{\mathbf{A}}$ at $(x, t)$ implies the non-properness of $\pi_{i}$ restricted to $\Delta_{i}^{\mathbf{A}}$ at $x$.

The fact that the restriction of $\Pi_{i}$ to $W_{i}^{\mathbf{A}}$ is birational comes immediately from the fact that the restriction of $\pi_{i}$ to $\Delta_{i}^{\mathbf{A}}$ is birational.

This ends the proof of Proposition 3

We are now ready to state our main geometric result which characterizes the set of generalized critical values of $f$. 


\subsection{Main geometric result}

The combination of Proposition 2, Proposition 3 and Lemma 2] leads to the following result.

Theorem 3 (Geometric characterization of generalized critical values) There exists a Zariski-closed subset $\mathcal{A} \subsetneq G L_{n}(\mathbb{C})$ such that for all $\mathbf{A} \in G L_{n}(\mathbb{Q}) \backslash \mathcal{A}$ the set $K_{\infty}(f)$ of asymptotic critical values of $f$ is contained in the set of non-properness of the projection $\pi_{T}$ restricted to the Zariski-closure of the constructible set defined by:

$$
f^{\mathbf{A}}-T=\frac{\partial f^{\mathbf{A}}}{\partial X_{2}}=\cdots=\frac{\partial f^{\mathbf{A}}}{\partial X_{n}}=0, \quad \frac{\partial f^{\mathbf{A}}}{\partial X_{1}} \neq 0 .
$$

Remark 3 Remark that the above result only states that $K_{\infty}(f)$ is contained in the set of non-properness $\mathcal{Z}$ of the projection $\Pi:\left(x_{1}, \ldots, x_{n}, t\right) \in \mathbb{C}^{n+1} \rightarrow t \in \mathbb{C}$ restricted to $W_{1}$. The latter set is zero-dimensional (see [24]). Nevertheless, this inclusion can be strict since some points in $\mathcal{Z}$ can depend on $\mathbf{A}$.

Example 3 In [40], the authors use [24, Lemma 3.10] to compute the set of non-properness of a projection restricted to an algebraic variety. Denoting by $I^{\mathbf{A}}$ the ideal associated to $W_{1}^{\mathbf{A}}$, this algorithm specializes in our case to computing the characteristic polynomial of the multiplication by $X_{1}$ in $\mathbb{Q}(T)\left[X_{1}, \ldots, X_{n}\right] / I^{\mathbf{A}}$. The set of non-properness of the projection on $T$ is the reunion of the zero-sets of the denominators of this characteristic polynomial seen as univariate in $X_{1}$.

Consider the polynomial which is already studied in Section 0

$$
f=X_{1}+X_{1}^{2} X_{2}+X_{1}^{4} X_{2} X_{3}
$$

Performing the linear change of variables below

$$
\begin{aligned}
& X_{1} \leftarrow X_{1}+X_{2}+X_{3} \\
& X_{2} \leftarrow X_{1}+2 X_{2}+3 X_{3} \\
& X_{3} \leftarrow X_{1}+4 X_{2}+9 X_{3}
\end{aligned}
$$

one finds as a set of non-properness for the projection on $T$ the zero-set of the univariate polynomial below

$$
256 T^{2}(20 T+1)
$$

Performing the linear change of variables below

$$
\begin{aligned}
& X_{1} \leftarrow 10213 X_{1}+41543 X_{2}+51532 X_{3} \\
& X_{2} \leftarrow X_{1}+44904 X_{2}+10334 X_{3} \\
& X_{3} \leftarrow X_{1}+58200 X_{2}+1597 X_{3}
\end{aligned}
$$

one finds as a set of non-properness for the projection on $T$ the zero-set of the univariate polynomial below

$$
T^{2}(898540 T+117941) .
$$

Thus $K_{\infty}(f)$ is the gcd of these univariate polynomials and is $\{0\}$. 


\section{The algorithm and its complexity}

Given $f \in \mathbb{Q}\left[X_{1}, \ldots, X_{n}\right]$, we show now how to compute the set of generalized critical values $K(f)$ of the mapping $x \in \mathbb{C}^{n} \rightarrow f(x) \in \mathbb{C}$. Since $K(f)=K_{0}(f) \cup K_{\infty}(f)$, we focus first on the computation of $K_{0}(f)$ and then we deal with the computation of $K_{\infty}(f)$.

Our algorithms rely on tools coming from polynomial system solving. We use Gröbner bases and the Geometric resolution algorithm. Gröbner bases are a standard tool in polynomial system solving since it allows to test the membership of a polynomial to an ideal, to compute elimination ideals, and to reduce the computation of rational parameterizations of the roots of a zero-dimensional ideal to linear algebra computations in a polynomial ring quotiented by the considered ideal. Gröbner bases have a complexity within $D^{\mathcal{O}(n)}$ arithmetic operations in $\mathbb{Q}$ when the input polynomial family generates a zero-dimensional ideal (see [30]).

The geometric resolution algorithm [20, 31] is more recent and goes back to [19, 17, 18]. The input is a polynomial system of equation and inequations encoded by a straight-line program and defining a constructible set. It returns generic points in each equi-dimensional component of the Zariski-closure of the constructible set defined by the input. These generic points are encoded by rational parameterizations

$$
\left\{\begin{array}{ccc}
X_{n} & = & \frac{q_{n}(T)}{q_{0}(T)} \\
& \vdots \\
X_{1} & = & \frac{q_{1}(T)}{q_{0}(T)} \\
q(T) & = & 0
\end{array}\right.
$$

where $T$ is a new variable. Thus the output of the geometric resolution algorithm is a list of $n+2$-tuples of univariate polynomials $\left(q, q_{0}, q_{1}, \ldots, q_{n}\right)$. This algorithm is probabilistic, but its complexity is well-controlled. We denote by $M(x)$ the cost of multiplying univariate polynomials of degree $x$ and the notation $p \in \mathcal{O}_{\log }(x)$ means that $p \in \mathcal{O}\left(x \log x^{a}\right)$ for some constant $a$.

Theorem 4 (Complexity result for geometric resolution) [31] Let $g_{1}, \ldots, g_{S}$ and $g$ be polynomials of degree bounded by $D$ in $\mathbb{Q}\left[X_{1}, \ldots, X_{n}\right]$, represented by a Straight-Line Program of length $\mathcal{L}$. There exists an algorithm computing a geometric resolution of the Zariski-closure $V\left(g_{1}, \ldots, g_{S}\right) \backslash V(g)$ whose arithmetic complexity is:

$$
\mathcal{O}_{\log }\left(S n^{4}\left(n \mathcal{L}+n^{4}\right) M(D \mathfrak{d})\right)^{3}
$$

where $\mathfrak{d}$ is the maximum of the sums of the algebraic degrees of the irreducible components of the intermediate varieties defined as the Zariski-closures of the constructible sets $g_{1}=\cdots=$ $g_{i}=0, g \neq 0$ for $i$ in $1, \ldots, S$.

Remark 4 In [31], the author proves that the bit complexity of his algorithm is

$$
\tau \mathcal{O}_{\log }\left(S n^{4}\left(n \mathcal{L}+n^{4}\right) M(D \mathfrak{d})\right)^{4}
$$


where $\tau$ bounds the bit-size of the coefficients of the input polynomial system.

In practice, Gröbner bases remain, in general, the fastest tool to solve polynomial systems, in particular when the algorithms [14, 15] are used. The geometric resolution algorithm is implemented as a Magma package by G. Lecerf (see [29]).

Hereafter, we describe how to compute $K_{0}(f)$ and $K_{\infty}(f)$ using Gröbner bases and the geometric resolution algorithm. When using Gröbner bases, one obtains a deterministic algorithm and an efficient behaviour in practice (see Section 17). When using the geometric resolution algorithm, we obtain a probabilistic algorithm whose complexity is well-controlled.

Computation of $K_{0}(f)$. The first step of an algorithm computing $K(f)$ is obviously the computation of the set of critical values $K_{0}(f)$ of $f$. This is encoded as the set of roots of a univariate polynomial. Denote by $I$ the ideal

$$
\left\langle f-T, \frac{\partial f}{\partial X_{1}}, \ldots, \frac{\partial f}{\partial X_{n}}\right\rangle .
$$

Sard's Theorem ensures that there exists $P \in \mathbb{Q}[T]$ such that: $\langle P\rangle=I \cap \mathbb{Q}[T]$ and, by definition, the set of roots of $P$ is $K_{0}(f)$.

Gröbner bases allow such computations of elimination ideals.

$$
\begin{array}{||l||}
\hline \text { Algorithm computing } K_{0}(f) \text { using Gröbner bases } \\
\hline \text { - Input: a polynomial } f \text { in } \mathbb{Q}\left[X_{1}, \ldots, X_{n}\right] \text {. } \\
\text { - Output: a univariate polynomial } P \in \mathbb{Q}[T] \text { such that its } \\
\text { zero-set is } K_{0}(f) \text {. } \\
\text { - Compute a Gröbner basis } G \text { for an elimination ordering } \\
{\left[X_{1}, \ldots, X_{n}\right]>[T] \text { of the ideal generated by: }} \\
\qquad\left\langle f-T, \frac{\partial f}{\partial X_{1}}, \ldots, \frac{\partial f}{\partial X_{n}}\right\rangle . \\
\text { - Return the element of } G \text { belonging to } \mathbb{Q}[T] .
\end{array}
$$

Remark that $\sharp K_{0}(f) \leq(D-1)^{n}$ since it is defined as the values taken by a polynomial on each isolated primary component of an ideal defined by $n$ polynomials of degree $D-1$. So, one could expect to obtain an algorithm computing $K_{0}(f)$ having a complexity within $(D-1)^{\mathcal{O}(n)}$. This aim can be reached by using the geometric resolution Algorithm. The first step is the computation of rational parameterizations of generic points in each equidimensional component of the algebraic variety defined by:

$$
\frac{\partial f}{\partial X_{1}}=\cdots=\frac{\partial f}{\partial X_{n}}=0 .
$$


Once they are obtained, one can obtain the values taken by $f$ at these points which are encoded by a univariate polynomial.

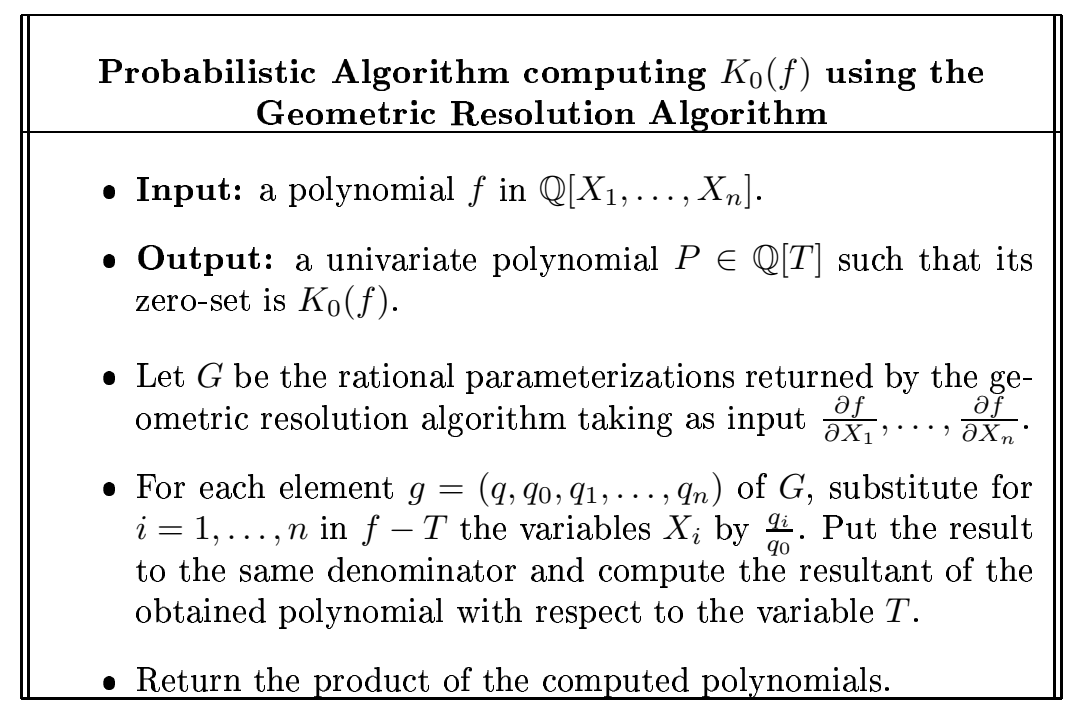

The complexity of the above algorithm is dominated by the cost of computing a geometric resolution of the algebraic variety defined by:

$$
\frac{\partial f}{\partial X_{1}}=\cdots=\frac{\partial f}{\partial X_{n}}=0
$$

Computation of $K_{\infty}(f)$. It remains to show how to compute $K_{\infty}(f)$. Following Remark 3 and Example 3 this task can be achieved by linear algebra computations in the quotient ring $\mathbb{Q}(T)\left[X_{1}, \ldots, X_{n}\right] / I^{\mathbf{A}}$ where $I^{\mathbf{A}}$ is the ideal associated to $W_{1}^{\mathbf{A}}$.

Deterministic Algorithm. In order to obtain a deterministic algorithm, we must check that the chosen linear change of variables $\mathbf{A}$ is generic enough. Given $f \in \mathbb{Q}\left[X_{1}, \ldots, X_{n}\right]$, denote by $\operatorname{deg}\left(f,\left[X_{1}, \ldots, X_{i}\right]\right)$ the degree of $f$ when it is seen as a polynomial in $\mathbb{Q}\left(X_{i+2}, \ldots, X_{n}\right)\left[X_{1}, \ldots, X_{i}\right]$ and denote by $\phi_{i}$ the mapping sending $f \in \mathbb{Q}\left[X_{1}, \ldots, X_{n}\right]$ to $X_{0}^{\operatorname{deg}\left(f,\left[X_{1}, \ldots, X_{i+1}\right]\right)} f\left(\frac{X_{1}}{X_{0}}, \ldots, \frac{X_{i+1}}{X_{0}}, X_{i+2}, \ldots, X_{n}\right)$.

From [40, 28], the properness of $\Pi_{i}$ restricted to the Zariski-closure of

$$
f^{\mathbf{A}}-T=\frac{\partial f^{\mathbf{A}}}{\partial X_{1}}=\cdots=\frac{\partial f^{\mathbf{A}}}{\partial X_{i}}, \quad \frac{f^{\mathbf{A}}}{\partial X_{i+1}} \neq 0
$$

can be tested by computing the intersection of the projective closure of $W_{n-i}^{\mathbf{A}}$ in $\mathbb{P}^{i+1}(\mathbb{C}) \times$ $\mathbb{C}^{n-i}$ and the hyperplane at infinity. This can be done by Gröbner bases computations (see [11]). A preliminary test consists in applying $\phi_{i}$ to the system defining $W$, instantiating 
$X_{0}$ to 1 and check that when substituting $X_{k}$ by 1 (for $k=1, \ldots, i-1$ ), the obtained polynomial system generates $\langle 1\rangle$. Using Gröbner bases, such computations are particularly efficient when the choice of $\mathbf{A}$ is a correct one. Modular computations can also be used to perform some preliminary tests on sparse matrices $\mathbf{A} \in G L_{n}(\mathbb{Q})$.

In the sequel we denote by Set0fNonProperness a subroutine taking as input a polynomial system of equations and inequations and a set of variables and computes the set of non-properness of the projection on the variables given as input restricted to the Zariskiclosure of the constructible set defined by the input polynomial system. Such a procedure is described in [40, 28].

$$
\begin{array}{||l||}
\hline \text { Algorithm computing } K_{\infty}(f) \text { using Gröbner bases } \\
\text { - Input: a polynomial } f \text { in } \mathbb{Q}\left[X_{1}, \ldots, X_{n}\right] . \\
\text { - Output: a univariate polynomial } P \in \mathbb{Q}[T] \text { such that its } \\
\text { zero-set is } K_{0}(f) \text {. } \\
\text { - Choose randomly } \mathbf{A} \in G L_{n}(\mathbb{C}) \text { and check if it is generic } \\
\text { enough until this test returns true. } \\
\text { - Return } \operatorname{SetOfNonProperness~}\left(\left[f^{\mathbf{A}}-T=\frac{\partial f^{\mathbf{A}}}{\partial X_{1}}=\cdots=\right.\right. \\
\\
\left.\left.\frac{\partial f^{\mathbf{A}}}{\partial X_{n-1}}=0, \frac{\partial f^{\mathbf{A}}}{\partial X_{n}} \neq 0\right],\{T\}\right)
\end{array}
$$

Probabilistic Algorithm. As in the case of the computation of $K_{0}(f)$, Gröbner bases do not allow to obtain complexity results even if the first choice of $\mathbf{A}$ is supposed to be correct. To reach this aim, one also uses extensions of the geometric resolution algorithms allowing to lift the parameter. Here, in the input polynomial system

$$
f^{\mathbf{A}}-T=\frac{\partial f^{\mathbf{A}}}{\partial X_{1}}=\cdots=\frac{\partial f^{\mathbf{A}}}{\partial X_{n-1}}=0, \frac{\partial f^{\mathbf{A}}}{\partial X_{n}} \neq 0
$$

$T$ is considered as the parameter. From [2], if $\mathbf{A}$ is generic enough, this defines a zerodimensional system generating a radical ideal in $\mathbb{Q}(T)\left[X_{1}, \ldots, X_{n}\right]$. The output is a geometric resolution

$$
\left\{\begin{array}{ccc}
X_{n} & = & \frac{q_{n}\left(X_{1}, T\right)}{q_{0}\left(X_{1}, T\right)} \\
& \vdots \\
X_{2} & = & \frac{q_{2}\left(X_{1}, T\right)}{q_{0}\left(X_{1}, T\right)} \\
q\left(X_{1}, T\right) & = & 0
\end{array}\right.
$$

The set of non properness of the projection on $T$ restricted to the Zariski-closure of the constructible set defined by the input polynomial system is contained the least commun multiple of the denominators of the coefficients of $q$. 


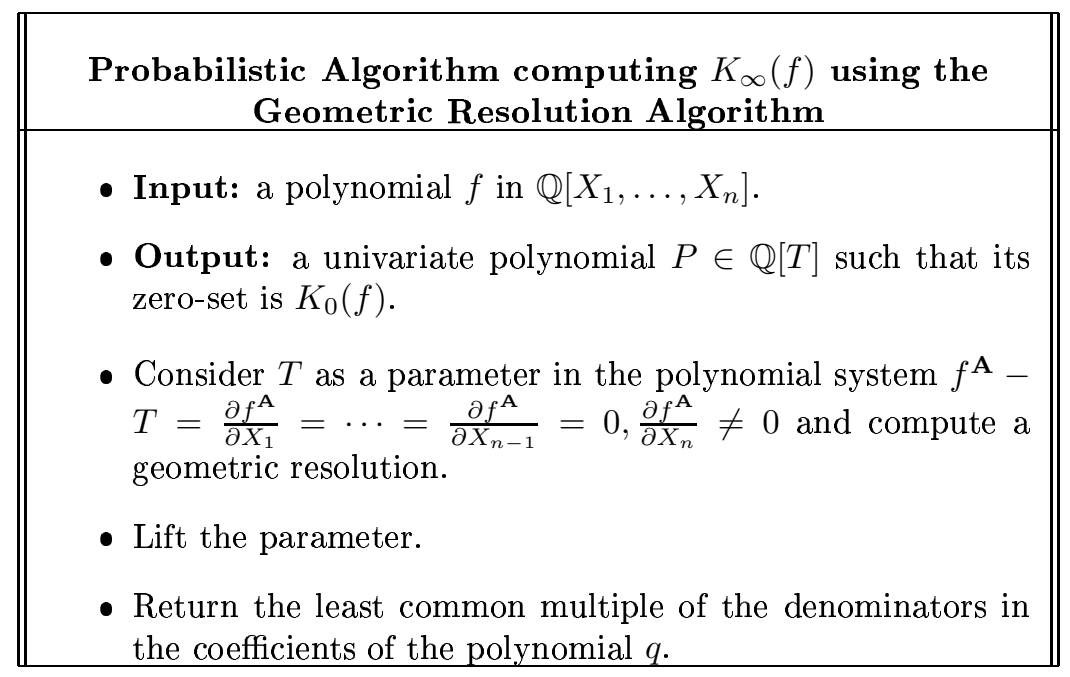

Complexity estimates. Using Theorem 4] (see [31]), the probabilistic versions of the algorithms computing $K_{0}(f)$ and $K_{\infty}(f)$ allow to perform a complexity analysis. Indeed, using strong versions of Bézout theorems (see [16]), the sum of the degrees of the primary components of the ideal generated by :

$$
\frac{\partial f}{X_{1}}=\cdots=\frac{\partial f}{X_{n}}=0
$$

is bounded by $(D-1)^{n}$ (where $D$ is the degree of $f$ ). Thus, the polynomial returned by the probabilistic algorithm computing $K_{0}(f)$ has a degree bounded by $(D-1)^{n}$.

We focus now on the computation of $K_{\infty}(f)$. Our algorithm computed a polynomial encoding the set of non-properness of a projection restricted to the curve defined as the Zariski-closure of the solution set:

$$
f^{\mathbf{A}}-T=\frac{\partial f^{\mathbf{A}}}{\partial X_{1}}=\cdots=\frac{\partial f^{\mathbf{A}}}{\partial X_{n-1}}, \quad \frac{\partial f^{\mathbf{A}}}{\partial X_{n}} \neq 0
$$

which has a degree bounded by $(D-1)^{n-1}$ since, from Bézout's theorem the Zariski-closure of the complex solution set of .

$$
f^{\mathbf{A}}-T=\frac{\partial f^{\mathbf{A}}}{\partial X_{1}}=\cdots=\frac{\partial f^{\mathbf{A}}}{\partial X_{n-1}}, \quad \frac{\partial f^{\mathbf{A}}}{\partial X_{n}} \neq 0
$$

has degree at most $(D-1)^{n-1}$. From [43, the lifting of the parameter $T$ has a complexity which is log-linear in the evaluation complexity of the above system and quadratic in the degree of the studied curve.

Bounding the evaluation complexity of $f$ by $D^{n}$, this discussion leads to the following complexity result. 
Theorem 5 (Complexity result) The above probabilistic algorithm computing $K_{0}(f)$ performs at most $\mathcal{O}\left(n^{7} D^{4 n}\right)$ arithmetic operations in $\mathbb{Q}$.

The above probabilistic algorithm computing $K_{\infty}(f)$ performs at most $\mathcal{O}\left(n^{7} D^{4 n}\right)$ arithmetic operations in $\mathbb{Q}$.

Remark 5 Using Remark 4, the bit-complexity of the probabilistic versions of our algorithms is $\mathcal{O}\left(\tau n^{7} D^{5 n}\right)$ where $\tau$ bounds the bit-size of the coefficients in $f$.

\section{Application I: testing the emptiness of a semi-algebraic set defined by a single inequality}

In this section, we show how to use the above algorithm to compute at least one point in each connected component of a semi-algebraic set defined by a single inequality.

This result can be proved using classical techniques of real algebraic geometry.

Theorem 6 (Semi-algebraic sets) Let $f$ be a polynomial in $\mathbb{Q}\left[X_{1}, \ldots, X_{n}\right]$ and $\mathcal{S}$ be the semi-algebraic set defined by $f>0$. Let $e \in \mathbb{Q}$ be such that $0<e<\min (|r|, r \in K(f) \cap \mathbb{R})$.

Consider the hypersurface $\mathcal{H}_{e}$ defined by $f-e=0$. Then, for each connected component $S$ of $\mathcal{S}$, there exists a connected component $C$ of $\mathcal{H}_{e} \cap \mathbb{R}^{n}$ such that $C \subset S$.

Proof. Let $\varepsilon$ be an infinitesimal and $\mathcal{H}_{\varepsilon} \subset \mathbb{C}\langle\varepsilon\rangle^{n}$ be the hypersurface defined by $f-\varepsilon=0$. From the intermediate value theorem, each connected component $S$ contains a point $x_{S}$ such that $f\left(x_{S}\right)=\varepsilon$. The connected component $C_{x_{S}} \subset \mathbb{R}\langle\varepsilon\rangle^{n}$ of $\mathcal{H}_{\varepsilon} \cap \mathbb{R}\langle\varepsilon\rangle^{n}$ is contained in $S$ since $f$ does not vanish on $C_{x_{S}}$.

From the transfer principle, this implies that there exist $e_{0}>0$ such that for all $0<e^{\prime}<$ $e_{0}$ and for all connected component $S$ of $\mathcal{S}$ there exists a connected component $C_{e^{\prime}}$ of the real locus of the hypersurface defined by $f-e^{\prime}=0$ such that $C_{e^{\prime}} \subset S$. Consider such a rational number $e^{\prime}$ and a positive rational number $e$ such that $0<e<\min (|r|, r \in K(f))$. We prove now that there exists a connected component $C_{e}$ of the real locus of the hypersurface defined by $f-e=0$ such that $C_{e} \subset S$ for all connected component $S$ of $\mathcal{S}$.

Suppose that $e^{\prime}$ is chosen small enough such that $\left.K(f) \cap\right] 0, e^{\prime}\left[=\emptyset\right.$. If $0<e<e^{\prime}$, the assertion follows immediately.

Suppose now that $e>e^{\prime}$. In [32, the authors prove that $f$ realizes a locally trivial fibration on $\mathbb{R}^{n} \backslash f^{-1}(K(f))$. This implies that there exists a diffeomorphism $\varphi$ such that, for all $\left.e_{1} \in\right] e^{\prime}, e[$, denoting by $\pi$ the projection on the second member of the cartesian product $\left.f^{-1}\left(e_{1}\right) \times\right] e, e^{\prime}[$ the following diagram is commutative

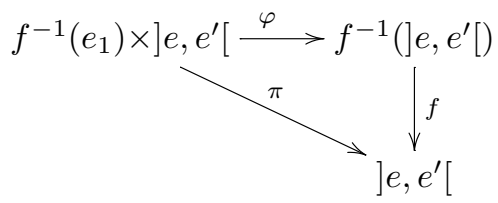


This implies that one can link any point $x_{e^{\prime}}$ of $C_{e^{\prime}}$ to a point $x_{e}$ in $\mathcal{H}_{e} \cap \mathbb{R}^{n}$ via a continuous path on which $f$ does not vanish. Then, $x_{e}$ belongs to $S$ and if $C_{e}$ denotes the connected component of $\mathcal{H}_{e} \cap \mathbb{R}^{n}$ containing $x_{e}$, one has $C_{e} \subset S$ since $f$ is constant on $C_{e}$.

Remark 6 From Theorem [6, deciding the emptiness of the semi-algebraic set defined by $f>0$ is reduced to decide if a hypersurface defined by a polynomial with coefficients in $\mathbb{Q}$ contains real points.

Substituting $f$ by $-f$ one can deal with semi-algebraic sets defined by $f<0$. At last, computing at least one point in each connected component of the semi-algebraic set defined by $f \neq 0$ is done by computing at least one point in each connected component of the semialgebraic sets defined by $f>0$ and $f<0$.

The Algorithm. The algorithm relies on Theorem[ Given a polynomial $f$ in $\mathbb{Q}\left[X_{1}, \ldots, X_{n}\right]$ of degree $D$, the algorithm computes at least one point in each connected component of the semi-algebraic set defined by $f>0$. The first step is the computation of the set of generalized critical values of the mapping $f: x \in \mathbb{C}^{n} \rightarrow f(x) \in \mathbb{C}$. Using the probabilistic version of the algorithm provided in Section 4 this can be done within $\mathcal{O}\left(n^{7} D^{4 n}\right)$ arithmetic operations in $\mathbb{Q}$.

We have seen in the preceeding section that the degree of the polynomials encoding generalized critical values is bounded by $\mathcal{O}\left(D^{n}\right)$. Thus, isolating the real solutions of the polynomial encoding the set of generalized critical values of $f$ is done within $\mathcal{O}\left(D^{3 n}\right)$ arithmetic operations in $\mathbb{Q}$ using the variant of Uspensky's algorithm designed in [37]. Choosing a positive rational number $e$ between 0 and the smallest positive real generalized critical value is immediate.

It remains to compute at least one point in each connected component of the real counterpart of the hypersurface defined by $f-e=0$. This can be done using the algorithm designed in [39] within $\mathcal{O}\left(n^{7} D^{3 n}\right)$ arithmetic operations in $\mathbb{Q}$. This algorithm is based on computations of critical loci of generic projections. This leads to the following theorem.

Theorem 7 (Complexity result) Let $f$ be a polynomial in $\mathbb{Q}\left[X_{1}, \ldots, X_{n}\right]$ of degree $D$ and $\mathcal{S}$ be the semi-algebraic set defined by $f>0$. The probabilistic version of the above algorithm computes at least one point in each connected component of $\mathcal{S}$ with a complexity within $\mathcal{O}\left(n^{7} D^{4 n}\right)$ arithmetic operations in $\mathbb{Q}$.

\section{Application II: determining the existence of real regu- lar points in a hypersurface}

In this section, we focus on the following problem: given a polynomial $f \in \mathbb{Q}\left[X_{1}\right.$, $\left.\ldots, X_{n}\right]$ of degree $D$, decide if the hypersurface $\mathcal{H}$ defined by $f=0$ contains real regular points. Hence, the problem consists in deciding if the real dimension of $\mathcal{H} \cap \mathbb{R}^{n}$ equals the complex dimension of $\mathcal{H}$. This problem appears in many applications (in particular 
in automated geometric reasoning or in algorithmic geometry) studying generic geometric situations.

This can be solved using the Cylindrical Algebraic Decomposition but the complexity of this method is doubly exponential in the number of variables and, in practice, this method is limited to problems having 3 or 4 variables.

Such a problem can also be tackled by computing the real radical of the ideal $\langle f\rangle \subset$ $\mathbb{Q}\left[X_{1}, \ldots, X_{n}\right]$ (which is the radical ideal of $\mathbb{Q}\left[X_{1}, \ldots, X_{n}\right]$ whose associated algebraic variety is the smallest one - for the inclusion ordering - containing $\mathcal{H} \cap \mathbb{R}^{n}$ ). This can be done by using the algorithms designed in [8]. These algorithms perform a recursive study on imbricated singular loci of the studied varieties. Up to our knowledge, bounding the degree of the singular locus of a variety, the degree of the singular locus of the singular locus and so on yields doubly exponential bounds in the number of variables. Thus, the complexity of such methods seems to be doubly exponential in the number of variables and no efficient implementation have been obtained from these works.

The real dimension of $\mathcal{H}$ can be computed using [7, Chapter 14]. The complexity of this algorithm is $D^{\mathcal{O}\left(n^{2}\right)}$. Nevertheless, this algorithm does not provide satisfactory results in practice due to the use of several infinitesimals and some growth of degree which are difficult to manage in practical implementations and lead to a high complexity constant (which is here as an exponent).

All the methods above compute exactly the real dimension of $\mathcal{H} \cap \mathbb{R}^{n}$ which is stronger than the expected output. In the case where $f$ is square-free, the problem in which we are interested can be tackled by deciding if all the semi-algebraic sets $\mathcal{S}_{i} \subset \mathbb{R}^{n}$ defined by $f=0, \frac{\partial f}{\partial X_{i}} \neq 0$ (for $i=1, \ldots, n$ ) are empty or not. Each semi-algebraic set $\mathcal{S}_{i}$ is studied by studying the real algebraic sets of $\mathbb{R}\langle\varepsilon\rangle^{n}$ defined by $f=\frac{\partial f}{\partial X_{i}}-\varepsilon=0$ and $f=\frac{\partial f}{\partial X_{i}}+\varepsilon=0$. The complexity of this method is $D^{\mathcal{O}(n)}$ but we are lead here to study $n$ distinct semi-algebraic sets defined by an equation (of degree $D$ ) and an inequation (of degree $D-1$ ).

In the sequel, we show how to reduce the problem of determining the existence of real regular points in a hypersurface defined by $f=0$ to the problem of deciding if there exist $\left(x, x^{\prime}\right) \in \mathbb{R}^{n} \times \mathbb{R}^{n}$ such that $f(x)>0$ and $f\left(x^{\prime}\right)<0$. The probabilistic version of our algorithm has a complexity within $\mathcal{O}\left(n^{7} D^{4 n}\right)$ arithmetic operations in $\mathbb{Q}$.

Theorem 8 (Existence of regular real points) Let $f$ be a square-free polynomial in $\mathbb{Q}\left[X_{1}, \ldots, X_{n}\right]$ and $\mathcal{H} \subset \mathbb{C}^{n}$ be the hypersurface defined by $f=0$. There exist regular real points in $\mathcal{H}$ if and only if there exist $\left(x, x^{\prime}\right) \in \mathbb{R}^{n} \times \mathbb{R}^{n}$ such that $f(x)>0$ and $f\left(x^{\prime}\right)<0$.

Proof. Suppose first that $\mathcal{H}$ contains real regular points and let $y$ be such a point. Since $f$ is square-free, one has $\operatorname{grad}_{y}(f) \neq \mathbf{0}$. Now, considering the line passing through $y$ and supported by the vector $\operatorname{grad}_{y}(f)$ and a Taylor development of $f$ along this line near $y$, it is clear that $f$ is positive and negative along this line.

Suppose now that $\mathcal{H}$ does not contain a real regular zero. Then, the real locus of $\mathcal{H}$ (which may be empty) is contained in the singular locus of $\mathcal{H}$. Since the co-dimension of the singular locus of $\mathcal{H}$ is greater than 1 , the complementary of $\mathcal{H} \cap \mathbb{R}^{n}$ in $\mathbb{R}^{n}$ is connected. This 
implies that either the semi-algebraic set defined by $f>0$ is empty or the semi-algebraic set defined by $f<0$ is empty.

The Algorithm. The algorithm based on Theorem 8 works as follows. The input of the algorithm is a polynomial $f$ in $\mathbb{Q}\left[X_{1}, \ldots, X_{n}\right]$ of degree $D$. Compute the square-free part of $f$.

Determine the sign of $f$ on a randomly chosen point at which $f$ does not vanish. In practice, this step is immediate while in theory, on has to test each point in a grid of size $D^{n^{2}}$ to be sure to find a point at which $f$ does not vanish. Since our complexity estimates are based on probabilistic algorithms, we suppose that the cost of this step is the one of the evaluation of $f$, i.e. $\mathcal{O}_{\log }\left(D^{n}\right)$ arithmetic operations in $\mathbb{Q}$.

If $f$ is found to be positive on the test-point, test the emptiness of the semi-algebraic defined by $f<0$, else test the emptiness of the semi-algebraic set defined by $f>0$. Using the algorithm designed in Section [5 using the computation of generalized critical values, this is done within $\mathcal{O}\left(n^{7} D^{4 n}\right)$ arithmetic operations in $\mathbb{Q}$.

\section{Practical results}

We have implemented the algorithms presented in Sections 4 , 5 and 6 using Gröbner bases.

The Gröbner engine which is used is FGB [13] which is implemented in C by J.-C. Faugère. Computing rational parametrizations of the complex roots of a zero-dimensional ideal from a Gröbner basis is done by RS which is implemented in C by F. Rouillier. Isolation of real roots of univariate polynomials with rational coefficients is done by RS using the algorithm provided in [34].

The resulting implementation is a part of the development version of the RAGLiB Maple library [38]. We do not describe implementation details allowing us to avoid an explicit linear change of variables by using a choice of generic projections. We also don't describe modular tests which allow us to test if the chosen projections are good. However, observe that the first choices have always been correct.

All the computations have been performed on a PC Intel Pentium Centrino Processor $1.86 \mathrm{GHz}$ with 2048 Kbytes of Cache and $1024 \mathrm{MB}$ of RAM.

\subsection{Description of the test-suite.}

The following polynomial appears in a problem of algorithmic geometry studying the Voronoi Diagram of three lines in $\mathbb{R}^{3}$. In [12], the authors focus on determining topology changes of the Voronoi diagram of three lines in $\mathbb{R}^{3}$. The question was first reduced to determining if the zero-set of discriminant of the following polynomial with respect of the variable $u$ contains real regular points. This discriminant has degree 30. This discriminant is the product of a polynomial of degree 18 and several polynomials up to an odd power whom zero-set could not contain a real regular point since they are sums of squares. The polynomial of degree 
18 is Lazard II. D. Lazard and S. Lazard have also asked to determine if the following polynomial which is denoted by Lazard $\mathbf{I}$ in the sequel is always positive.

$$
\begin{array}{r}
16 a^{2}\left(\alpha^{2}+1+\beta^{2}\right) u^{4}+16 a\left(-\alpha \beta a^{2}+a x \alpha+2 a \alpha^{2}+2 a+2 a \beta^{2}+a y \beta-\alpha \beta\right) u^{3}+ \\
\left(\left(24 a^{2}+4 a^{4}\right) \alpha^{2}+\left(-24 \beta a^{3}-24 a \beta-8 y a^{3}+24 x a^{2}-8 a y\right) \alpha+24 a^{2} \beta^{2}+4 \beta^{2}-\right. \\
\left.8 \beta x a^{3}+4 y^{2} a^{2}+24 y \beta a^{2}-8 a x \beta+16 a^{2}+4 x^{2} a^{2}\right) u^{2}+\left(-4 \alpha a^{3}+4 y a^{2}-\right. \\
\left.4 a x-8 a \alpha+8 \beta a^{2}+4 \beta\right)(\beta-a \alpha+y-a x) u+\left(a^{2}+1\right)(\beta-a \alpha+y-a x)^{2}
\end{array}
$$

In the sequel, we denote by Lazard I the above polynomial and by Lazard II the discriminant of Lazard I with respect to the variable $u$.

The following polynomial appears in [27]. The problem consists in determining the conditions on $a, b, c$ and $d$ such that the ellipse defined by:

$$
\frac{(x-c)^{2}}{a^{2}}+\frac{(y-d)^{2}}{b^{2}}=1
$$

is inside the circle defined by $x^{2}+y^{2}-1=0$.

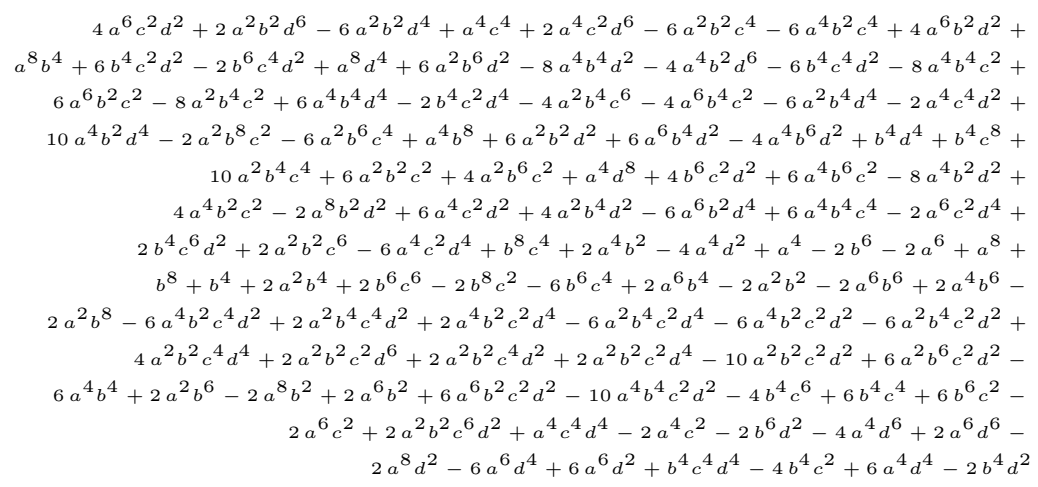

Below, in the column JK we give the timings for computing generalized critical values by using the algorithm of [32. We obviously use the same Gröbner engine FGb than ours for this algorithm. The column AlgoHyp corresponds to the maximum of the timings obtained by

- our algorithm computing at least one point in each connected component of the semialgebraic set defined by the positivity of our input;

- our algorithm computing at least one point in each connected component of the semialgebraic set defined by the negativity of our input.

The column CAD contains the timings of an implementation of the open CAD algorithm in Maple which is due to G. Moroz and F. Rouillier. It outputs a set of rational points in each cell homeomorphic to $] 0,1{ }^{n}$ (where $n$ is the number of variables) of a CAD adapted 
to the input polynomial. The symbol $\infty$ means that the computations have been stopped after 2 days of computations without getting a result.

The algorithms provided in [7] never end on these examples. The practical behaviour on this test-suite illustrates what happens in most of the examples we studied: on problems having at most 4 variables, the open CAD algorithm behaves well (except on polynomials having a big degree) and our implementation has comparable timings even if it is sometimes slower. On problems having more variables, our implementation ends with reasonnable timings while open CAD does not end after 2 days of computations. This is mainly due to the highest degrees appearing in the projection step of CAD while the degrees of the polynomials appearing during the execution of our algorithms is better controlled. Note also that our algorithm is now implemented using a Gröbner basis engine which can be strongly improved for problems having 3 or 4 variables. In these situations, we expect to obtain strong improvements. At last, remark that the generic choice of projections to compute generalized critical values induces a growth of coefficients which reduces the practical performances of our contribution. We plan now to investigate how to compute generalized critical values without any change of variables. This could strongly speed up our contribution. That's why that we are convinced that our method is a promising one.

\begin{tabular}{||c|c|c|c|c|c||}
\hline Pbm & $\sharp$ vars & Degree & JK & AlgoHyp & CAD \\
\hline Lazard I & 6 & 8 & $\infty$ & 60 sec. & $\infty$ \\
\hline Lazard II & 5 & 18 & $\infty$ & 10 hours. & $\infty$ \\
\hline Ellips-Circle & 4 & 12 & $\infty$ & 90 sec. & 5 min. \\
\hline
\end{tabular}

\section{References}

[1] P. Aubry, F. Rouillier, and M. Safey El Din. Real solving for positive dimensional systems. Journal of Symbolic Computation, 34(6):543-560, 2002.

[2] B. Bank, M. Giusti, J. Heintz, and G.-M. Mbakop. Polar varieties and efficient real equation solving: the hypersurface case. Journal of Complexity, 13(1):5-27, 1997.

[3] B. Bank, M. Giusti, J. Heintz, and L.-M. Pardo. Generalized polar varieties and efficient real elimination procedure. Kybernetika, 40(5):519-550, 2004.

[4] B. Bank, M. Giusti, J. Heintz, and L.-M. Pardo. Generalized polar varieties: Geometry and algorithms. to appear in Journal of complexity, 2005.

[5] S. Basu, R. Pollack, and M.-F. Roy. On the combinatorial and algebraic complexity of quantifier elimination. Journal of ACM, 43(6):1002-1045, 1996.

[6] S. Basu, R. Pollack, and M.-F. Roy. A new algorithm to find a point in every cell defined by a family of polynomials. In Quantifier elimination and cylindrical algebraic decomposition. Springer-Verlag, 1998. 
[7] S. Basu, R. Pollack, and M.-F. Roy. Algorithms in real algebraic geometry. SpringerVerlag, 2003.

[8] E. Becker and R. Neuhaus Computation of real radicals for polynomial ideals. Computational Algebraic Geometry, Vol. 109 of Progress in Mathematics, pp. 1-20, Birkhaüser, 1993.

[9] R. Benedetti, J.-J. Risler Real algebraic and semi-algebraic sets. Actualités Mathématiques, Hermann, 1990.

[10] G.E. Collins, Quantifier elimination for real closed fields by cylindrical algebraic decomposition. Lecture notes in computer science, 33:515-532, 1975.

[11] D. Cox, J. Little, and D. O'Shea. Ideals, varieties and algorithms : an introduction to computational algebraic geometry and commutative algebra. Springer-Verlag, 1992.

[12] H. Everett, D. Lazard, S. Lazard, and M. Safey El Din. The topology of the Voronoi diagram of three lines in $\mathbb{R}^{3}$. In preparation.

[13] J.-C. Faugère. Gb/FGb. Available at http://fgbrs.lip6.fr.

[14] J.-C. Faugère. A new efficient algorithm for computing Gröbner bases (F4).-. Journal of Pure and Applied Algebra, 139(1-3):61-88, 1999.

[15] J.-C. Faugère. A new efficient algorithm for computing Gröbner without reduction to zero (F5). In Proceedings of ISSAC 2002, pages 75 - 83. ACM Press, 2002.

[16] W. Fulton, Intersection Theory. Ergebnisse der Mathematik und ihrer Grenzgebiete, 2. Springer-Verlag, 1984.

[17] M. Giusti, K. Hägele, J. Heintz, J.-E Morais, J.-L. Montaña, and L.-M. Pardo. Lower bounds for Diophantine approximation. In Proceedings of MEGA'96, number 117, 118 in Journal of Pure and Applied Algebra, pages 277-317, 1997.

[18] M. Giusti, J. Heintz, J.-E. Morais, J. Morgenstern, and L.-M. Pardo. Straight-line programs in geometric elimination theory. Journal of Pure and Applied Algebra, 124:101$146,1998$.

[19] M. Giusti, J. Heintz, J.-E. Morais, and L.-M. Pardo. When polynomial equation systems can be solved fast? In Proceedings of AAECC-11, volume 948 of LNCS, pages 205-231. Springer, 1995.

[20] M. Giusti, G. Lecerf, and B. Salvy. A Gröbner free alternative for polynomial system solving. Journal of Complexity, 17(1):154-211, 2001.

[21] D. Grigoriev and N. Vorobjov. Solving systems of polynomials inequalities in subexponential time. Journal of Symbolic Computation, 5:37-64, 1988. 
[22] J. Heintz, M.-F. Roy, and P. Solernò. On the complexity of semi-algebraic sets. In Proceedings IFIP'89 San Francisco, North-Holland, 1989.

[23] J. Heintz, M.-F. Roy, and P. Solernò. On the theoretical and practical complexity of the existential theory of the reals. The Computer Journal, 36(5):427-431, 1993.

[24] Z. Jelonek. Testing sets for properness of polynomial mappings. Mathematische Annalen, 315(1):1-35, 1999.

[25] Z. Jelonek. Topological characterization of finite mappings. Bull. Polish Acad. Sci. Math., 49(3):279-283, 2001.

[26] Z. Jelonek, K. Kurdyka. On asymptotic critical values of a complex polynomial. Journal für die Reine und Angewandte Mathematik, 565:1-11, 2003.

[27] D. Lazard. Quantifier elimination: optimal solution for two classical examples. Journal of Symbolic Computation, 1988.

[28] D. Lazard and F. Rouillier. Solving parametric polynomial systems. Technical report, INRIA, 2004.

[29] G. Lecerf. Kronecker magma package for solving polynomial systems. available at http://www.math.uvsq.fr/ lecerf/software/.

[30] Y.N. Lakshman. A single exponential bound of the complexity of computing Gröbner bases of zero-dimensional ideals. In C. Traverso T. Mora, editor, Proc. Effective Methods in Algebraic Geometry, MEGA, vol. 94 of Progress in Mathematics, pages 227-234. Birkhäuser, 1991.

[31] G. Lecerf. Computing the equidimensional decomposition of an algebraic closed set by means of lifting fibers. Journal of Complexity, 19(4):564-596, 2003.

[32] K. Kurdyka, P. Orro, S. Simon, Semi-algebraic Sard's theorem for generalized critical values. Journal of Differentiable Geometry 56 (2000), 67-92.

[33] J. Renegar. On the computational complexity and geometry of the first-order theory of the reals. Journal of Symbolic Computation, 13(3):255-352, 1992.

[34] F. Rouillier. RS, RealSolving. available at http://fgbrs.lip6.fr.

[35] F. Rouillier. Solving zero-dimensional systems through the Rational Univariate Representation. AAECC Journal, 9(5):433-461, 1999.

[36] F. Rouillier, M.-F. Roy, and M. Safey El Din. Finding at least one point in each connected component of a real algebraic set defined by a single equation. Journal of Complexity, 16:716-750, 2000. 
[37] F. Rouillier and P. Zimmermann. Efficient isolation of polynomial real roots. Journal of Computational and Applied Mathematics, 162(1):33-50, 2003.

[38] M. Safey El Din. RAGLib (Real Algebraic Geometry Library). available at http://www-calfor.lip6.fr/ safey/RAGLib, 2003.

[39] M. Safey El Din and É. Schost. Polar varieties and computation of one point in each connected component of a smooth real algebraic set. In Proceedings of the 2003 international symposium on Symbolic and algebraic computation, pages 224-231. ACM Press, 2003.

[40] M. Safey El Din and É. Schost. Properness defects of projections and computation of one point in each connected component of a real algebraic set. Journal of Discrete and Computational Geometry, 32(3):417-430, 2004.

[41] M. Safey El Din, Finding sampling points on real hypersurfaces is easier in singular situations. Electronic proceedings of MEGA, 2005.

[42] M. Safey El Din, Generalized critical values and solving polynomial inequalities. Proceedings of International Conference on Polynomial Systems (2004).

[43] E. Schost, Computing Parametric Geometric Resolutions. Journal of Applicable Algebra in Engineering, Communication and Computing, 13(5): 349 - 393, 2003.

[44] I. Shafarevich, Basic Algebraic Geometry I, Springer-Verlag, (1977). 


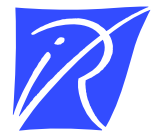

Unité de recherche INRIA Rocquencourt

Domaine de Voluceau - Rocquencourt - BP 105 - 78153 Le Chesnay Cedex (France)

Unité de recherche INRIA Futurs : Parc Club Orsay Université - ZAC des Vignes

4, rue Jacques Monod - 91893 ORSAY Cedex (France)

Unité de recherche INRIA Lorraine : LORIA, Technopôle de Nancy-Brabois - Campus scientifique

615, rue du Jardin Botanique - BP 101 - 54602 Villers-lès-Nancy Cedex (France)

Unité de recherche INRIA Rennes : IRISA, Campus universitaire de Beaulieu - 35042 Rennes Cedex (France)

Unité de recherche INRIA Rhône-Alpes : 655, avenue de l'Europe - 38334 Montbonnot Saint-Ismier (France)

Unité de recherche INRIA Sophia Antipolis : 2004, route des Lucioles - BP 93 - 06902 Sophia Antipolis Cedex (France)

INRIA - Domaine de Voluceau - Rocquencourt, BP 105 - 78153 Le Chesnay Cedex (France)

http://www.inria.fr

ISSN 0249-6399 